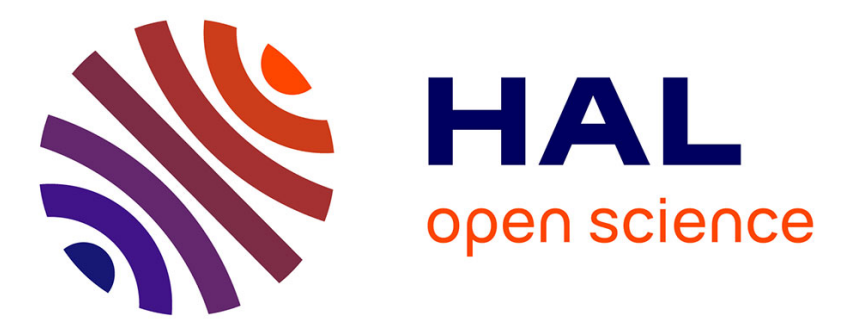

\title{
On the use of tracking loops for low-complexity multi-path channel estimation in OFDM systems
}

Huaqiang Shu, Eric Pierre Simon, Laurent Ros

\section{To cite this version:}

Huaqiang Shu, Eric Pierre Simon, Laurent Ros. On the use of tracking loops for low-complexity multi-path channel estimation in OFDM systems. Signal Processing, 2015, 117, pp.174-187. 10.1016/j.sigpro.2015.05.012 . hal-01174265

\section{HAL Id: hal-01174265 \\ https://hal.science/hal-01174265}

Submitted on 8 Jul 2015

HAL is a multi-disciplinary open access archive for the deposit and dissemination of scientific research documents, whether they are published or not. The documents may come from teaching and research institutions in France or abroad, or from public or private research centers.
L'archive ouverte pluridisciplinaire HAL, est destinée au dépôt et à la diffusion de documents scientifiques de niveau recherche, publiés ou non, émanant des établissements d'enseignement et de recherche français ou étrangers, des laboratoires publics ou privés. 


\title{
On the Use of Tracking Loops for Low-Complexity Multi-Path Channel Estimation in OFDM Systems *
}

\author{
Huaqiang Shu $\dagger^{\dagger}$ Eric Pierre Simon $\stackrel{\ddagger}{\ddagger}$ and Laurent Ros ${ }^{\dagger}$
}

\begin{abstract}
This paper treats pilot aided multi-path channel estimation with tracking loops for OFDM systems under slow to moderate fading conditions. Recent works have presented theoretical results for the tuning of second-order and thirdorder tracking loops in the particular context of Jakes's Doppler spectrum channel. The method for getting the loop coefficients resorted either to the use of a given constraint, which made the obtained coefficients sub-optimal, or was obtained in part by simulations. Here, we perform a global optimization of the coefficients without constraints to get the optimal coefficients, and analytical formulas are provided. One remarkable result of this optimization is that only the natural frequency depends on the transmission parameters, i.e., the channel Doppler spectrum, the power delay profile, and the noise variance. Consequently, only one parameter has to be tuned. Moreover, asymptotic performance is formulated in a more general way as a function of the $2 r$ th moments of the Doppler spectrum ( $r$ is the loop order). Hence, all our derivations are usable for any Doppler spectrum and are not specific to Jakes's Doppler spectrum. A complete table sums up for the three orders the theoretical results of the optimal coefficients together with the asymptotic performance. The performance is also compared with that of the asymptotic Kalman filter.
\end{abstract}

Index Terms-OFDM, Channel estimation, Kalman filter, Phase-locked loop

\section{INTRODUCTION}

This paper treats pilot aided multi-path channel estimation for orthogonal frequency division multiplexing (OFDM) transmissions under slow to moderate fading conditions. In the OFDM technique, a wide-band frequency-selective channel is converted to a number of parallel narrow-band flat fading subchannels that are free of inter-symbol interference and inter-carrier interference (for negligible channel time variations within one OFDM symbol period $T$ ). However, reliable estimation of the channel is crucial in order to recover the information symbols. Most of the conventional channel estimation methods work with a symbol-by-symbol scheme [2][4], using the correlation of the channel only in the frequency domain, i.e., the correlation between the subchannels. More advanced algorithms also exploit the time-domain correlation of the channel, and various approaches are possible, as reviewed in [5]. In this paper, we focus on the class of parametric estimators that track the complex amplitudes (CAs) of the paths of the channel, assuming a primary acquisition of the delays. Also, we assume moderate normalized Doppler frequency

\footnotetext{
* Part of this work was presented in the conference ISCCSP 2014 [1]

†Univ. Grenoble Alpes, GIPSA-Lab, F-38000 Grenoble, France; CNRS, GIPSA-Lab, F-38000 Grenoble, France

${ }^{\ddagger}$ IEMN lab, TELICE group, 59655 Villeneuve d’Ascq, France.
}

( $f_{d} T$ ) values, i.e., $f_{d} T \leq 10^{-2}$, whereby the channel variation within the duration of one symbol can be neglected (as in [4], [6]-[11]). Note that this scenario typically corresponds to low mobility with the classical standards, like LTE or WiMax, but could also correspond to high mobility since low carrier frequencies are investigated with the development of cognitive radio. For instance, VHF/UHF television broadcast bands from $54 \mathrm{MHz}$ to $862 \mathrm{MHz}$ [12] and aeronautical bands from 960 $\mathrm{MHz}$ to $1215 \mathrm{MHz}$ are planned for deployment. For such systems, $f_{d} T$ values around $10^{-2}$ can correspond to several hundreds of $\mathrm{km} / \mathrm{h}$.

A natural way to perform a joint estimation of the multipath CAs is to use Kalman filters (KFs). In this perspective, a linear recursive state space model, such as the $r$ th order autoregressive model, $\mathrm{AR} r$, or the $r$ th order integrated Random Walk model, RWr [13], has to be used to approximate the dynamics of the the channel CAs (see [5]). AR model Kalman filters are widely used in OFDM systems [6], [7], [14], [15], based on a correlation matching (CM) criterion to fix the AR1 coefficient. To pursuit a more precise estimate, especially in slow to moderate fading conditions, the asymptotic variance (MAV) criterion has been proved to be more efficient [16][18], associated to either the AR model or the RW model. Recent research in [13], [19] has shown analytically (for a singlecarrier single path Rayleigh channel with Jakes's Doppler Spectrum scenario) that the Asymptotic Mean Squared Error (AMSE) performance of KFs can be improved by switching from a first-order model, e.g., AR1 or RW1, to a secondorder RW or a third-order RW model. The resulting RW2$\mathrm{KF}$ and RW3-KF have been shown to better take into account the fact that the exact path CA continues in a given direction for several symbols if $f_{d} T$ is low, and then shows a strong trend behavior. The larger the order of the model, the better the approximation of the actual fading statistics, but also the greater the computational complexity.

However, the KFs for the joint estimation of the path CAs in OFDM are very complicated. We now focus on a reduced-complexity adaptive algorithm based on a Complex Amplitude Tracking Loop (CATL), and referred to here as $\mathrm{RW} r$-LS-CATL. It can be interpreted as a simplification of the reference $\mathrm{RW} r-\mathrm{KF}$, for the same model orders $r=1,2$ or 3. The RW2-LS-CATL algorithm was introduced in [20], then theoretically analyzed in [5]. Such an algorithm is generally slower than a second-order KF during the convergence, but shows almost the same asymptotic performance (e.g., the performance obtained in tracking mode). This algorithm is 
inspired by digital Phase-Locked Loops (PLLs), as well as by the "prediction-correction" principle of the KF in the steady-state mode [21], [22], given the close link between the two [23], [24]. As its main advantage, the RWr-LS-CATL presents a linear complexity in terms of the number of pilot sub-carriers $N_{p}$ versus a cubic complexity $\left(O\left(N_{p}^{3}\right)\right)$ for the reference Kalman algorithms (details in Section III).

Inspired by digital PLL, the RWr-LS-CATL is based on a CATL structure, and on a specific CA error signal. This error signal is created from the LS estimate of the path's CA obtained from the pilot subcarriers of the current OFDM symbol. The key ideas that allow interpreting the RWr-LSCATL as a simplified algorithm compared to RW $r$-KF rely on two main points: its per-path processing, and its steady-state mode. First, the front-end pilot-aided LS estimator permits converting the primary observation at pilot frequencies into a primary (instantaneous) estimate of the path's CAs, and then obtaining separate error signals for each of the $L$ paths. The use of the LS estimator allows us to have in output a perpath processing with $L$ branches that track each path CA separately, instead of the full joint processing as in the highly complicated RWr-KF algorithm. Consequently, the per-path processing gives a first reduction in complexity with a $\mathrm{RW} r$ LS-CATL that acts in each branch $l$, i.e., for each multi-path component $\alpha^{(l)}$, as a single-path single-carrier RWr-CATL [24]. Secondly, the single-path single-carrier RW $r$-CATLs used in each branch can be viewed as steady-state versions of the single-path single-carrier RW $r$-KFs. Then, we gain here a second reduction in complexity compared to the Kalman-based algorithms, since the single-path single-carrier RWr-CATLs are constant coefficient filters, unlike the single-path singlecarrier $\mathrm{RW} r$-KFs, which require matrix inversion at each iteration to update their time-varying coefficients. However, we can expect almost the same AMSE performance since [13] has proved that the single-path single-carrier $\mathrm{RW} r-\mathrm{KF}$ is equivalent in steady-state mode and slow-tracking scenario to the single-path single-carrier RWr-CATL with fixed given parameters (specified in [13]).

The contribution of this paper is multi-fold, as an extension of our previous studies that were restricted either to the second-order loops and the Rayleigh channel with Jakes's Doppler spectrum, or to the single-carrier single-path scenario. In this paper we perform and present for the first time a global optimization of the $r$ coefficients of the RWr-CATL in order to minimize the AMSE. Note that in our previous paper, [24], dedicated to single path single carrier systems, the method of getting the loop coefficients for the third-order loop resorted to the use of a given constraint (see [24], Eq. (65) or appendix C), which made the obtained coefficients sub-optimal. And in the previous paper, [5], dedicated to the second-order loop $(r=2)$, we optimized analytically only one parameter, the natural frequency of the loop $f_{n}$, and the other parameter (the damping factor $\zeta$ ) was fixed (at $\frac{1}{2}$ ) only by means of a gridsearch numerical optimization (see [5], figure 3). Note that part of our previous studies is used as a preliminary to this paper. A table summarizing these previous results is provided (see Table I) so that the interested reader can go back to the original developments. In this table, the global optimization means the optimization obtained without constraint.

One remarkable result of the present paper is the analytic proof that despite a global optimization, only one optimal parameter, the natural frequency $f_{n}$, depends on the transmission parameters, i.e., the channel Doppler spectrum, the power delay profile, and the noise variance. Consequently, only this parameter $f_{n}$ has to be tuned, even if we have a structure with $r=2$ or 3 parameters. In other words, the remaining $r-1$ parameters can be fixed to the optimal values delivered in this paper, regardless of the channel condition. This study provides then a complete table of the analytic formulae for the tuning and steady-state performance of the RW $r$-LS-CATL $(r=1,2,3)$, usable for the multi-path multicarrier scenario and adaptable to any Doppler spectrum model of a wide-sense stationary channel. Indeed, the asymptotic performance is formulated in a more general way than in our previous papers, as a function of the $2 r$ th moments of Doppler spectrum, where $r$ is the loop order. Hence, all our derivations are usable for any Doppler spectrum and are not specific to Jakes's Doppler spectrum as in our previous papers. For example, a 3D scattering model is more suitable than the Jakes model for an urban environment [26]. So we apply our formula for the 3D Doppler spectrum, and check their validity by means of simulations. In the same way, another contribution of the paper is the generalization to the second and third order loop of the link between the maximum frequencies of the Jakes and 3D model Doppler spectra that give the same asymptotic performance. This link could be obtained directly from the literature for the first order loop for which the performance depends on the second-order moment of the Doppler spectrum. Indeed it was shown in [27] that the second-order moment of the Jakes channel model and a 3D (flat spectrum) model could be matched by adapting the maximum Doppler frequency. We have thus extended these previous results to the fourth- and sixth-order moments, which can be exploited to calculate the AMSE of the RW2- and RW3-LS-CATL. A last contribution of this paper is a performance comparison between the AMSE of the tracking loops and that of the Kalman-based channel estimator for OFDM recently proposed in the literature [28] [29].

It is noteworthy that the RWr-LS-CATL refers to the multipath multi-carrier context whereas the RWr-CATL refers to the single-path single-carrier context. This paper is organized as follows: Section II presents the model. Section III gives the per-path presentation of the RW-LS-CATL, and a summary table containing the loop characteristics, the analytic formulae for the tuning, as well as the optimized performances. Finally, Section IV presents some simulation results and conclusions.

Notation: $[\mathbf{x}]_{k}$ denotes the $k$ th entry of the vector $\mathbf{x}$, and $[\mathbf{X}]_{m, n}$ denotes the $[m, n]$ th entry of the matrix $\mathbf{X}$ (indices begin from 1). The notation $\operatorname{diag}\{\mathbf{x}\}$ denotes the diagonal matrix with $\mathbf{x}$ on its main diagonal; $\operatorname{blkdiag}\{\mathbf{X}, \mathbf{Y}\}$ is the block diagonal matrix with the matrices $\mathbf{X}$ and $\mathbf{Y}$ on its main diagonal. $\mathbf{I}_{N}$ is the $N \times N$ identity matrix. The symbols $\{\cdot\}^{T}$, $\{\cdot\}^{H}$ and $\otimes$ stand for the transpose, Hermitian conjugate, and Kronecker product, respectively. 
TABLE I: Relevant works from the authors (or the same group) on similar topics on CATL (AMSE performance with $f_{d} T \ll 1$, link with asymptotic KF)

\begin{tabular}{|c|c|c|c|c|c|c|}
\hline Paper & $\begin{array}{l}\text { algo } \\
\text { type }\end{array}$ & $\begin{array}{l}\text { model } \\
\text { / order }\end{array}$ & $\begin{array}{r}\text { modulation } \\
\text { / channel }\end{array}$ & Doppler & $\begin{array}{r}\text { optimization } \\
\text { / type }\end{array}$ & comments \\
\hline $\begin{array}{c}{[20]} \\
\text { (conf.) }\end{array}$ & CATL & $\begin{array}{l}\mathrm{RW} \\
1,2\end{array}$ & $\begin{array}{r}\text { OFDM } \\
\text { multipath }\end{array}$ & Jakes & $\begin{array}{r}\text { global } \\
\text { numeric }\end{array}$ & $\begin{array}{r}\text { CATL 1st proposition, } \\
\text { no AMSE formula }\end{array}$ \\
\hline $\begin{array}{c}{[5]} \\
\text { (journal) }\end{array}$ & CATL & $\begin{array}{r}\mathrm{RW} \\
1,2\end{array}$ & $\begin{array}{r}\text { OFDM } \\
\text { multipath }\end{array}$ & Jakes & $\begin{array}{r}\text { global } \\
\text { theory/numeric }\end{array}$ & $\begin{array}{r}f_{n} T_{\text {(Jakes) }}: \text { theory } \\
\zeta: \text { numeric }\end{array}$ \\
\hline $\begin{array}{c}{[19]} \\
\text { (conf.) }\end{array}$ & KF & $\begin{array}{r}\mathrm{RW} \\
2\end{array}$ & $\begin{array}{r}\text { single-carrier } \\
\text { single-path }\end{array}$ & Jakes & $\begin{array}{l}\text { global } \\
\text { theory }\end{array}$ & $\begin{array}{r}\left.\text { asympt. RW2-KF (var. } \sigma_{u}^{2}\right) \\
\text { seen as CATL }\left(\zeta=\frac{\sqrt{2}}{2},\right. \\
\left.f_{n} T \approx \frac{1}{2 \pi}\left(\frac{\sigma_{u}}{\sigma_{L S}}\right)^{\frac{1}{2}}\right)\end{array}$ \\
\hline $\begin{array}{c}{[25]} \\
\text { (conf.) }\end{array}$ & CATL & $\begin{array}{r}\text { PLL } \\
3\end{array}$ & $\begin{array}{r}\text { single-carrier } \\
\text { single-path }\end{array}$ & Jakes & $\begin{array}{r}\text { constrained } \\
\text { theory/numeric }\end{array}$ & $\begin{array}{r}f_{n} T_{(\text {Jakes) }} \text { : theory } \\
m \approx 3, \zeta \approx 0.37 \text { : numeric }\end{array}$ \\
\hline $\begin{array}{c}{[24]} \\
\text { (journal) }\end{array}$ & CATL & $\begin{array}{r}\mathrm{RW} \\
3\end{array}$ & $\begin{array}{r}\text { single-carrier } \\
\text { single-path }\end{array}$ & Jakes & $\begin{array}{r}\text { constrained } \\
\text { theory }\end{array}$ & $\begin{array}{l}f_{n} T_{\text {(Jakes) }}, m \approx 3.19, \\
\quad \zeta \approx 0.39: \text { theory }\end{array}$ \\
\hline $\begin{array}{c}{[13]} \\
\text { (journal) }\end{array}$ & $\mathrm{KF}$ & $\begin{array}{r}\mathrm{RW} \\
3\end{array}$ & $\begin{array}{r}\text { single-carrier } \\
\text { single-path }\end{array}$ & $\begin{array}{r}\text { General, } \\
\text { Jakes }\end{array}$ & $\begin{array}{l}\text { global } \\
\text { theory }\end{array}$ & $\begin{array}{r}\text { asympt. RW3-KF }\left(\text { var. } \sigma_{u}^{2}\right) \\
\text { seen as CATL }(m=2, \\
\left.\zeta=\frac{1}{2}, f_{n} T \approx \frac{1}{2 \pi}\left(\frac{\sigma_{u}}{\sigma_{L S}}\right)^{\frac{1}{3}}\right)\end{array}$ \\
\hline $\begin{array}{l}\text { present } \\
\text { paper }\end{array}$ & CATL & $\begin{array}{r}\mathrm{RW} \\
1,2,3\end{array}$ & $\begin{array}{r}\text { OFDM } \\
\text { multipath }\end{array}$ & $\begin{array}{r}\text { General, } \\
\text { Jakes, 3D }\end{array}$ & $\begin{array}{l}\text { global } \\
\text { theory }\end{array}$ & $\begin{array}{r}f_{n} T_{\text {(opt) }} \text { and AMSE w.r.t. } S_{\alpha}, \\
\zeta_{(\text {opt })}, m_{\text {(opt) }} \text { for a given } r\end{array}$ \\
\hline
\end{tabular}

\section{SySTEM MODEL AND ESTIMATION OBJECTIVE}

\section{A. OFDM transmission}

Let us consider an OFDM system with $N$ sub-carriers, and a cyclic prefix length $N_{g}$. The duration of an OFDM symbol is $T=N_{T} T_{s}$, where $T_{s}$ is the sampling time and $N_{T}=N+N_{g}$. Let $\mathbf{x}_{(k)}$ be the sequence of transmitted elementary symbols of the $k$ th OFDM symbol. The $n$th element $\left[\mathbf{x}_{(k)}\right]_{n}(n=$ $1, \ldots, N)$ is the phase-shift modulated ( $M$-PSK) or quadrature amplitude modulated ( $M$-QAM) symbol transmitted on the sub-carrier with index $n-1-\frac{N}{2}$. The sequence of transmitted symbols is assumed to be zero-mean and stationary with unit variance: $\mathrm{E}\left\{\left|\left[\mathbf{x}_{(k)}\right]_{n}\right|^{2}\right\}=1$. Then, a quasi-static channel is considered where the channel may vary from one OFDM symbol to the other, yet constant within one OFDM symbol. This corresponds to a low mobility scenario. Let us consider the following parametric channel impulse response with $L$ paths at the $k$ th OFDM symbol:

$$
h_{(k)}(\tau)=\sum_{l=1}^{L} \alpha_{(k)}^{(l)} \delta\left(\tau-\tau^{(l)} T_{s}\right)
$$

where $\alpha_{(k)}^{(l)}$ is the complex amplitude of path $l$ and $\tau^{(l)} T_{s}$ is the corresponding physical time delay $\left(\tau^{(l)}\right.$ is not necessarily an integer, but $\tau^{(l)}<N_{g}$ ). Note that our work is developed in the framework of parametric (physical) channel model with the assumption of primary acquisition of the path delays as in [9], [10], instead of the discrete-time equivalent channel framework in [29]-[32]. The variable $\alpha_{(k)}^{(l)}$ is supposed to be a narrow-band stationary process, with power spectral density (PSD) $\Gamma_{\alpha}(f)$ with support limited within $\pm f_{d}$. The $L$ individual elements of $\left\{\alpha_{(k)}^{(l)}\right\}(l=1, \ldots, L)$ are uncorrelated with one another, with global variance $\sigma_{\alpha}^{2}=\sum_{l=1}^{L} \sigma_{\alpha(l)}^{2}$. We assume in the present study that during one OFDM symbol time, the CA in each path can be considered as a constant. As a consequence, the $k$ th received OFDM symbol $\mathbf{y}_{(k)}$ after transmission over the multi-path channel and fast Fourier transform demodulation, is given by

$$
\mathbf{y}_{(k)}=\operatorname{diag}\{\tilde{\boldsymbol{h}}\} \cdot \mathbf{x}_{(k)}+\mathbf{w}_{(k)},
$$

where $\mathbf{w}_{(k)}$ is an $N \times 1$ zero-mean complex circular Gaussian noise vector with covariance matrix $\sigma_{\mathrm{w}}^{2} \mathbf{I}_{N}$ and $\tilde{\boldsymbol{h}}_{(k)}$ is a $N \times 1$ vector containing the frequency response of the channel for the $k$ th OFDM symbol, $H_{(k)}(f)$, evaluated at the subcarrier frequency positions $f=-\frac{N}{2} \cdot \Delta f+n \Delta f$, for $n=0, \ldots, N-1$, where $\Delta f=\frac{1}{N T_{s}}$ is the subcarrier spacing :

$$
\tilde{\boldsymbol{h}}_{(k)}=\left[H_{(k)}\left(-\frac{N / 2}{N T_{s}}\right), \ldots, H_{(k)}\left(\frac{N / 2-1}{N T_{s}}\right)\right]^{T}
$$

with:

$$
H_{(k)}(f)=\sum_{l=1}^{L} \alpha_{(k)}^{(l)} e^{-j 2 \pi f \tau^{(l)} T_{s}}
$$

The observation model (2), based on a $N \times N$ diagonal matrix $\mathbf{H}_{(k)}=\operatorname{diag}\left\{\tilde{\boldsymbol{h}}_{(k)}\right\}$ containing the channels elements, can be equivalently written by the following model (5), based on a $N \times N$ diagonal matrix $\operatorname{diag}\left\{\mathbf{x}_{(k)}\right\}$ containing the symbols :

$$
\mathbf{y}_{(k)}=\operatorname{diag}\left\{\mathbf{x}_{(k)}\right\} \cdot \tilde{\boldsymbol{h}}_{(k)}+\mathbf{w}_{(k)},
$$


The vector $\tilde{\boldsymbol{h}}_{(k)}$ containing the frequency response of the channel, with its elements given by

$$
\left[\tilde{\boldsymbol{h}}_{(k)}\right]_{n}=\sum_{l=1}^{L}\left[\alpha_{(k)}^{(l)} \cdot e^{-j 2 \pi\left(\frac{n-1}{N}-\frac{1}{2}\right) \tau^{(l)}}\right],
$$

can be rewritten by the inner product:

$$
\tilde{\boldsymbol{h}}_{(k)}=\mathbf{F} \boldsymbol{\alpha}_{(k)},
$$

where $\boldsymbol{\alpha}_{(k)}=\left[\begin{array}{lll}\alpha_{(k)}^{(1)} & \ldots & \alpha_{(k)}^{(L)}\end{array}\right]^{T}$ is an $L \times 1$ vector containing only the CAs, and $\mathbf{F}$ is an $N \times L$ Fourier matrix depending only on the delay distribution, with elements given by $[\mathbf{F}]_{n, l}=$ $e^{-j 2 \pi\left(\frac{n-1}{N}-\frac{1}{2}\right) \tau^{(l)}}$, for $n=1, \ldots, N, l=1, \ldots, L$.

Using (7) and (5), the observation model of (2) can be rewritten [9] as

$$
\mathbf{y}_{(k)}=\operatorname{diag}\left\{\mathbf{x}_{(k)}\right\} \mathbf{F} \boldsymbol{\alpha}_{(k)}+\mathbf{w}_{(k)} .
$$

The $N_{p}$ pilot sub-carriers are evenly inserted into the $N$ subcarriers at the positions $\mathcal{P}=\left\{n_{p} \mid n_{p}=(p-1) L_{f}+1, p=\right.$ $\left.1, \ldots, N_{p}\right\}$ with $L_{f}$ the distance between two adjacent pilots. The received pilot sub-carriers can be written as

$$
\mathbf{y}_{\mathbf{p}(k)}=\mathbf{X}_{(k)} \boldsymbol{\alpha}_{(k)}+\mathbf{w}_{\mathbf{p}(k)}
$$

with $\mathbf{X}_{(k)}=\operatorname{diag}\left\{\mathbf{x}_{\mathbf{p}(k)}\right\} \mathbf{F}_{\mathbf{p}}$. The $N_{p} \times 1$ vectors $\mathbf{x}_{\mathbf{p}}, \mathbf{y}_{\mathbf{p}}$ and $\mathbf{w}_{\mathbf{p}}$ correspond, respectively, to the sent data symbol, the received data symbol, and the channel noise on the pilot sub-carriers. The $N_{p} \times L$ matrix $\mathbf{F}_{\mathbf{p}}$ is the Fourier matrix of the pilot subcarriers, with elements given by $\left[\mathbf{F}_{\mathbf{p}}\right]_{n_{p}, l}=e^{-j 2 \pi\left(\frac{n_{p}-1}{N}-\frac{1}{2}\right) \tau^{(l)}}$, where $n_{p} \in \mathcal{P}$.

\section{B. Channel model}

First, we assume that the $L$ CAs $\alpha_{(k)}^{(l)}$ are independent wide-sense stationary zero-mean complex circular Gaussian processes, namely the Rayleigh channel model (due to the law of the modulus of the CA). Second, the wireless mobile channel could be modeled differently, using different correlation functions $\mathbf{R}_{\alpha^{(l)}}^{(q)}=\mathrm{E}\left[\alpha_{(k)}^{(l)} \alpha_{(k-q)}^{(l) H}\right]$ or, equivalently, a different Doppler PSD $\Gamma_{\alpha^{(l)}}(f)$.

a) "Rayleigh-Jakes" model: The "Rayleigh-Jakes" model [33] is the most accepted model, with correlation coefficients for the time-lag $q$ given by

$$
\mathbf{R}_{\alpha^{(l)}(\text { Jakes })}^{(q)}=\sigma_{\alpha^{(l)}}^{2} J_{0}\left(2 \pi f_{d} T q\right),
$$

where $J_{0}$ is the zeroth order Bessel function of the first kind. The channel has a ' $U$ '-shaped Doppler spectrum defined by

$$
\Gamma_{\alpha^{(l)}(\text { Jakes })}(f)=\left\{\begin{array}{ccc}
\frac{\sigma_{\alpha}^{2}(l)}{\pi f_{d} \sqrt{1-\left(\frac{f}{f_{d}}\right)^{2}}}, & \text { if } & |f|<f_{d} \\
0, & \text { if } & |f| \geq f_{d},
\end{array}\right.
$$

where $f_{d}$ is the maximum Doppler frequency. b) $3 D$ model: Besides, Clark et al. [26] find that, in an indoor environment, the 3D model can properly describe a real channel with a constant spectrum within the maximum Doppler frequency:

$$
\Gamma_{\alpha^{(l)}(3 D)}(f)=\left\{\begin{array}{ccc}
\frac{\sigma_{\alpha}^{2}(l)}{2 f_{d}} & \text { if } & |f| \leq f_{d}, \\
0 & \text { if } & |f|>f_{d},
\end{array}\right.
$$

with the correlation function defined by

$$
\mathbf{R}_{\alpha^{(l)}(3 D)}^{(q)}=\sigma_{\alpha^{(l)}}^{2} \operatorname{sinc}\left(2 f_{d} T q\right) .
$$

We will see that the results summarized in this paper are usable for all channels as long as the Doppler spectrum is known.

\section{Estimation objective}

For an estimator $\hat{\boldsymbol{\alpha}}_{(k)}$ of $\boldsymbol{\alpha}_{(k)}$, the AMSE (mean value per path) is defined by

$$
\sigma_{\epsilon}^{2}=\frac{1}{L} \cdot E\left\{\left(\boldsymbol{\alpha}_{(k)}-\hat{\boldsymbol{\alpha}}_{(k)}\right)^{H} \cdot\left(\boldsymbol{\alpha}_{(k)}-\hat{\boldsymbol{\alpha}}_{(k)}\right)\right\} .
$$

The estimation objective is that the AMSE $\sigma_{\epsilon}^{2}$ approach the Bayesian Cramer-Rao bound (BCRB). The on-line BCRB for the estimation of $\boldsymbol{\alpha}_{(k)}$ from the present and previous observations over a multi-path Rayleigh fading channel and OFDM modulation has been calculated by [34].

\section{Per-Path Structured RW-LS-CATL}

\section{A. Equations of the RW-LS-CATL}

Inspired by a PLL structure, the RWr-CATL is an $r$ th order loop used for tracking the CAs of a single-path (flat-fading) channel in a single carrier system. The derivation of the CATL structure from the KF was carried out in [24] for the singlepath single-carrier context. This can be extended, in the case of a multi-path channel in a multi-carrier system, to a vector structured loop by employing a vector error signal at the frontend of the main loop [5] [20]. The equations of the vector CATL algorithm are given below.

Error signal:

$$
\boldsymbol{v}_{\boldsymbol{\epsilon}(k)}=f\left\{\mathbf{y}_{\mathbf{p}(k)} ; \hat{\boldsymbol{\alpha}}_{(k \mid k-1)}\right\},
$$

Loop filter:

$$
\begin{aligned}
& \boldsymbol{v}_{\text {Lag1 } 1(k)}=\boldsymbol{v}_{\text {Lag1 }(k-1)}+\boldsymbol{v}_{\boldsymbol{\epsilon}(k)}, \\
& \boldsymbol{v}_{\text {Lag2 } 2(k)}=\boldsymbol{v}_{\text {Lag } 2(k-1)}+\boldsymbol{v}_{\text {Lag1 } 1(k)}, \\
& \boldsymbol{v}_{\mathbf{c}(k)}=\mu_{1} \boldsymbol{v}_{\boldsymbol{\epsilon}(k)}+\mu_{2} \boldsymbol{v}_{\text {Lag1 }(k)}+\mu_{3} \boldsymbol{v}_{\text {Lag } 2(k-1)},
\end{aligned}
$$

Numerically controlled generator:

$$
\hat{\boldsymbol{\alpha}}_{(k+1 \mid k)}=\hat{\boldsymbol{\alpha}}_{(k \mid k-1)}+\boldsymbol{v}_{\mathbf{c}(k)},
$$

Final estimation:

$$
\hat{\boldsymbol{\alpha}}_{(k \mid k)}=\hat{\boldsymbol{\alpha}}_{(k \mid k-1)}+\mu_{1} \boldsymbol{v}_{\boldsymbol{\epsilon}(k)} .
$$

Note that the error signal $\boldsymbol{v}_{\boldsymbol{\epsilon}(k)}$ in (15) is used to drive the loop; it is usually defined as a function $f\{\cdot\}$ of the current received pilot symbol $\mathbf{y}_{\mathbf{p}(k)}$ and the previous prediction $\hat{\boldsymbol{\alpha}}_{(k \mid k-1)}$. Different error signals are possible, but lead to differing performances [20]. 


\section{B. Error signal}

The error signal employed in the RW $r$-LS-CATL is defined by

$$
\begin{aligned}
\boldsymbol{v}_{\boldsymbol{\epsilon}(k)} & =\mathbf{G}_{(k)} \mathbf{y}_{\mathbf{p}(k)}-\hat{\boldsymbol{\alpha}}_{(k \mid k-1)} \\
& =\hat{\boldsymbol{\alpha}}_{\mathbf{L S}(k)}-\hat{\boldsymbol{\alpha}}_{(k \mid k-1)},
\end{aligned}
$$

with $\mathbf{G}_{(k)}=\left(\mathbf{X}_{(k)}^{H} \mathbf{X}_{(k)}\right)^{-1} \mathbf{X}_{(k)}^{H}$ the LS operator. The loop equation (15) is then replaced by (21).

By using (9) and the definition of $\mathbf{G}_{(k)}$, we have

$$
\hat{\boldsymbol{\alpha}}_{\mathbf{L S}(k)}=\boldsymbol{\alpha}_{(k)}+\mathbf{w}_{\mathbf{L S}(k)},
$$

with $\mathbf{w}_{\mathbf{L S}(k)}$ the input noise of the loop:

$$
\mathbf{w}_{\mathbf{L S}(k)}=\mathbf{G}_{(k)} \mathbf{w}_{\mathbf{p}(k)},
$$

with the covariance matrix

$$
E\left\{\mathbf{w}_{\mathbf{L S}(k)} \cdot \mathbf{w}_{\mathbf{L S}(k)}^{H}\right\}=\sigma_{\mathbf{w}}^{2} \cdot\left(\mathbf{F}_{\mathbf{p}}^{H} \mathbf{F}_{\mathbf{p}}\right)^{-1} .
$$

The input loop noise for the path $l$ is

$$
\sigma_{\mathbf{L S}^{(l)}}^{2}=\sigma_{\mathrm{w}}^{2}\left[\left(\mathbf{F}_{\mathbf{p}}^{H} \mathbf{F}_{\mathbf{p}}\right)^{-1}\right]_{l, l} .
$$

The average noise variance for a single path $\left(\sigma_{\mathrm{LS}}^{2}=\right.$ $\left.\frac{1}{L} \sum_{l=1}^{L} \sigma_{\mathbf{L S}}^{2(l)}\right)$ can be calculated to be

$$
\sigma_{\mathrm{Ls}}^{2}=\lambda \cdot \frac{\sigma_{\mathrm{w}}^{2}}{N_{p}}
$$

with $\lambda$ a normalizing factor $(\geq 1)$ defined by

$$
\lambda=\frac{N_{p}}{L} \cdot \text { Trace }\left\{\left(\mathbf{F}_{\mathbf{p}}^{H} \mathbf{F}_{\mathbf{p}}\right)^{-1}\right\} .
$$

Note that $\operatorname{Trace}\left\{\left(\mathbf{F}_{\mathbf{p}}^{H} \mathbf{F}_{\mathbf{p}}\right)^{-1}\right\}$ depends on the channel power delay profile. In general, $\lambda>1$, while in the special case that $\mathbf{F}_{\mathbf{p}}^{H} \mathbf{F}_{\mathbf{p}}$ is a diagonal matrix equal to $N_{p} \mathbf{I}_{L}$, one has $\lambda=1$. This occurs only when the path delays of channel $\tau^{(l)} T_{s}$ are multiples of the sampling time $T_{s}$, i.e., if the $\tau^{(l)}$ are integers (see [28], appendix A for more details).

According to (15), the error signal $\boldsymbol{v}_{\boldsymbol{\epsilon}(k)}$ is actually the difference between the noisy observation of the CA vector $\hat{\boldsymbol{\alpha}}_{\mathrm{LS}(k)}$ (see (22)) and the last prediction $\boldsymbol{\alpha}_{(k \mid k-1)}$. The role of the LS estimator is to produce a preliminary estimate of $\alpha$ from the pilot sub-carriers of the current OFDM symbol. Such an error signal remains co-linear with the prediction error $\boldsymbol{\alpha}_{(k)}-\hat{\boldsymbol{\alpha}}_{(k \mid k-1)}$, and is free from inter-path interference.

\section{Per-path presentation of the RW-LS-CATL}

The RW $r$-LS-CATL can thus be interpreted as a parallel structure that is in fact a juxtaposition of $L$ single-path single-carrier RW $r$-CATLs combined with an LS estimator, as illustrated in Fig. 1a. This figure gives a per-path structured presentation of the RW $r$-LS-CATL, with each branch corresponding to a scalar single path RWr-CATL (Fig. 1b). As we can see in the branch structure figure, the RWr-CATL is controlled by $r$ loop coefficients. For $r=3$, the algorithm is controlled by three real positive parameters $\mu_{1}, \mu_{2}$ and $\mu_{3}$, for $r=2, \mu_{3}=0$, and for $r=1, \mu_{2}=\mu_{3}=0$. We have also connected these three parameters with the ones used

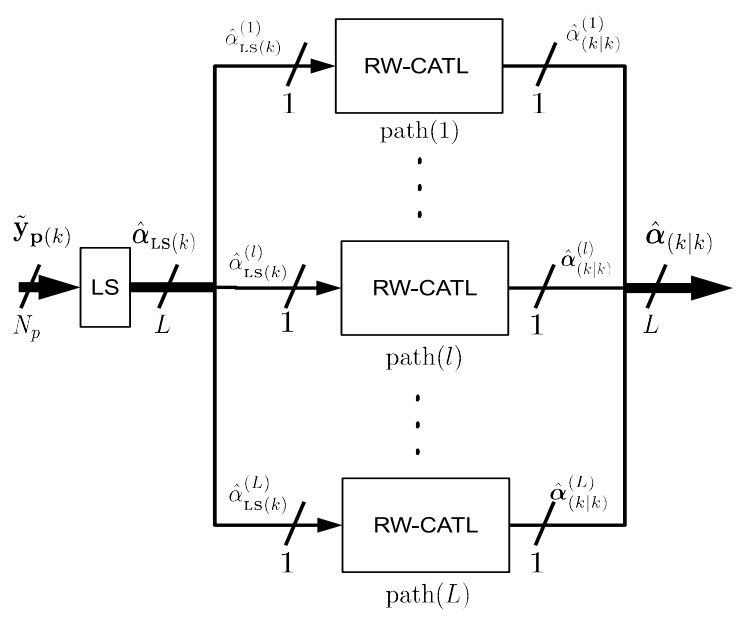

(a) global structure of the RW-LS-CATL

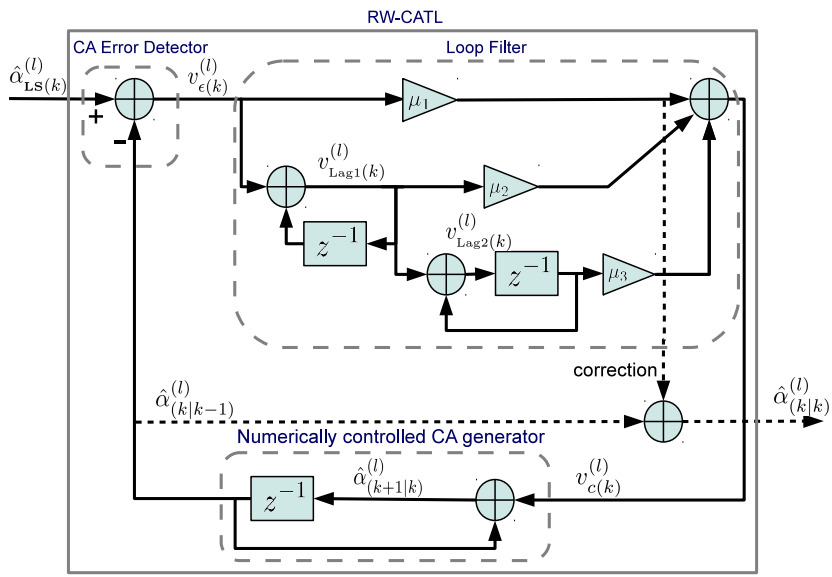

(b) RW-CATL structure of path $(l)$

Fig. 1: Presentation of the RW-LS-CATL

by a third-order PLL, the capacitance ratio $m$, the damping factor $\zeta$, and the natural frequency $f_{n}$ (or the natural impulsion $\omega_{n}=2 \pi f_{n}$ ). The relations between $\left[\mu_{1}, \mu_{2}\right]$ and $\left[\zeta, f_{n}\right]$ for the second-order loop, as well as between $\mu_{1}$ and the cut-off frequency $f_{n}$ are found for the first order, as summarized in Table II. Also, we would like to point out that, for a third order loop, the loop filter structure is not unique: the structure presented in $1 \mathrm{~b}$ is deduced from a RW3-KF; if the CATL is deduced directly from a third-order PLL, then the structure is slightly different, but can lead to the same AMSE variance, as shown in [25]. The analysis of the RW $r$-LS-CATL can be carried out by exploiting the results for the corresponding RW $r$-CATL.

\section{Asymptotic MSE of the RW-LS-CATL}

In single-path single-carrier scenarios, the asymptotic expressions of the RW-CATL were given in our previous paper [24]. These results can be applied to every branch (each one corresponds to a single path, according to (22)) of the RWLS-CATL by using the loop noise variance $\sigma_{\mathbf{L S}}^{2}(l)$ instead of the observation noise variance $\sigma_{\mathrm{w}}^{2}$ :

$$
\sigma_{\epsilon^{(l)}}^{2}=\sigma_{\epsilon \alpha^{(l)}}^{2}+\sigma_{\epsilon \mathrm{W}^{(l)}}^{2},
$$



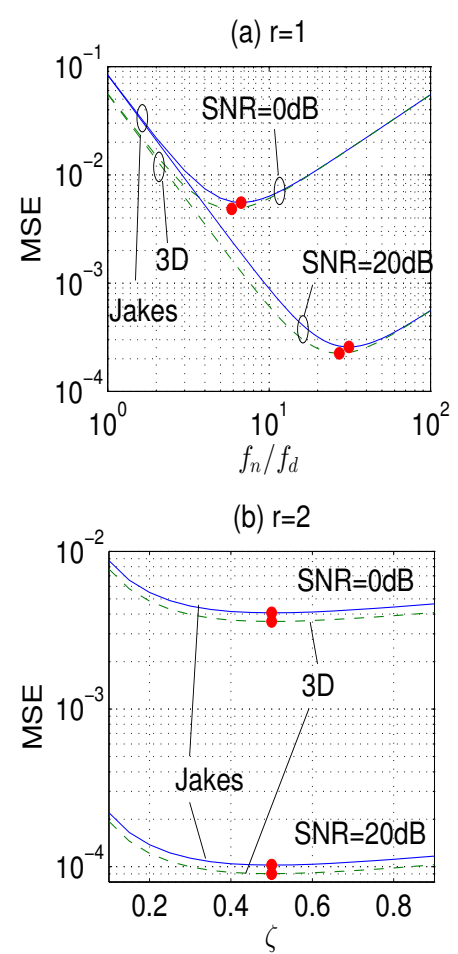
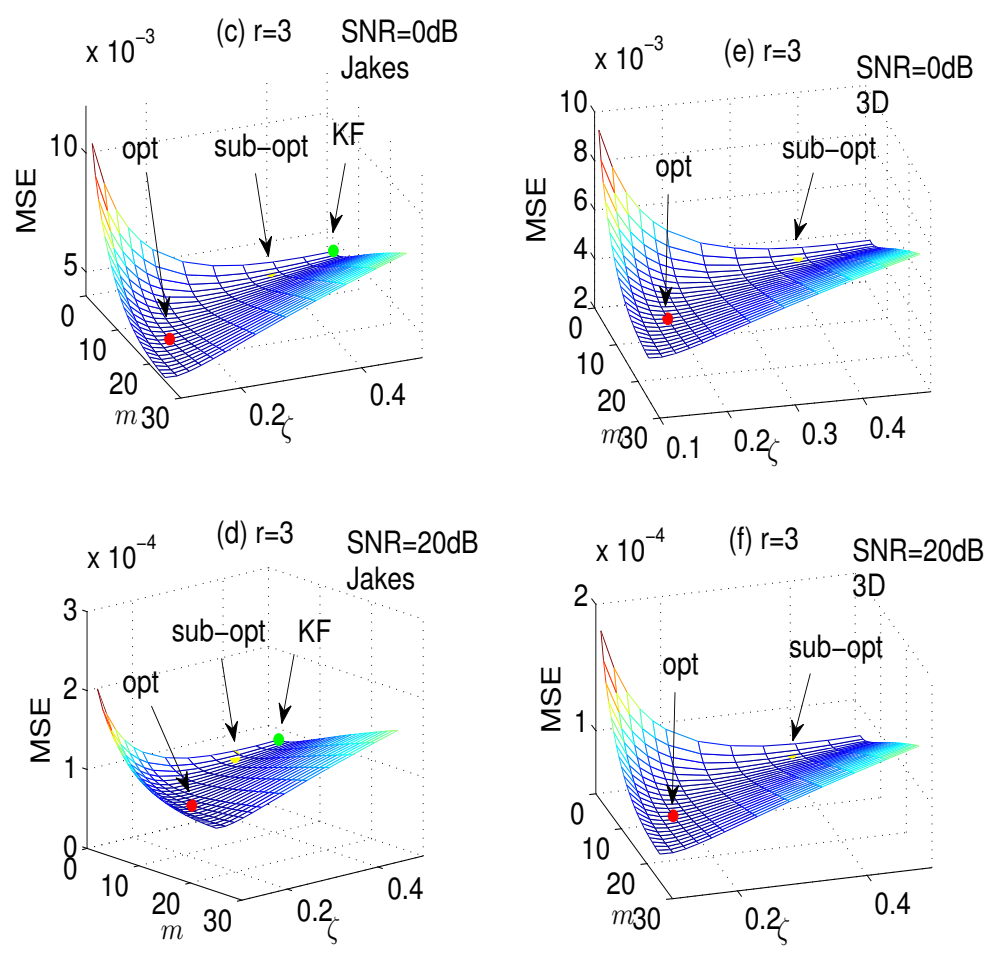

Fig. 2: AMSE versus loop parameters for different channel conditions (3D or Jakes Doppler, $\mathrm{SNR}=0$ or $20 \mathrm{~dB}$ ). (a) $r=1$, AMSE versus $f_{n} / f_{d}$; (b) $r=2$, AMSE versus $\zeta$; (c)-(f) $r=3$, AMSE versus $(m, \zeta)$

. Red points indicate the theoretical global optimum parameters. Black points indicate sub-optimum parameters with constraint (36). Green points indicate the equivalent optimized parameters for RW3-KF.

where $\sigma_{\epsilon \alpha^{(l)}}^{2}$, the dynamic error variance, results from the high-pass filtering of the input CA $\alpha_{(k)}$ while $\sigma_{\epsilon \mathrm{W}(l)}^{2}$, the static error variance, is the low-pass filtering of the input loop noise. We are interested in the global variance. Therefore, the variance of the estimation error is the average value of the two components:

$$
\sigma_{\epsilon}^{2}=\frac{1}{L} \sum_{l=1}^{L} \sigma_{\epsilon \alpha^{(l)}}^{2}+\frac{1}{L} \sum_{l=1}^{L} \sigma_{\epsilon \mathrm{W}^{(l)}}^{2}=\sigma_{\epsilon \alpha}^{2}+\sigma_{\epsilon \mathrm{W}}^{2} .
$$

The average dynamic error variance is calculated by the averaged spectrum:

$$
\sigma_{\epsilon \alpha}^{2}=\int_{-\frac{1}{2 T}}^{+\frac{1}{2 T}} \Gamma_{\alpha}(f) \cdot\left|1-\mathcal{L}\left(e^{j 2 \pi f T}\right)\right|^{2} d f,
$$

where $\Gamma_{\alpha}(f)=\frac{1}{L} \sum_{l=1}^{L} \Gamma_{\alpha^{(l)}}(f)$ and $\mathcal{L}(z)$ is the low-pass transfer function of the RW $r$-LS-CATL, expressed in the $z$ domain, or in the frequency-domain for $z=e^{j 2 \pi f T}$. The function $\mathcal{L}(z)$ is expressed in terms of the loop coefficient. To preserve a physical meaning, $\mathcal{L}\left(e^{s T}\right)$ in the $s$-domain (with $s=j 2 \pi f$ ) can also be approximated by the analog PLL transfer function $\tilde{\mathcal{L}}\left(e^{s T}\right)$. Note that the approximation $\mathcal{L}\left(e^{s T}\right) \approx \tilde{\mathcal{L}}\left(e^{s T}\right)$ is valid under the condition $f T \ll 1$, which yields $e^{-s T} \approx 1-s T$. The exact transfer function $\mathcal{L}(z)$ and the approximated one $\tilde{\mathcal{L}}\left(e^{s T}\right)$ are given in Table II (first part). Fig 3 shows the magnitude-frequency diagram of $\mathcal{L}(z), 1-\mathcal{L}(z)$ as well as $\tilde{\mathcal{L}}\left(e^{s T}\right)$ for the RW $r$-LS-CATL.

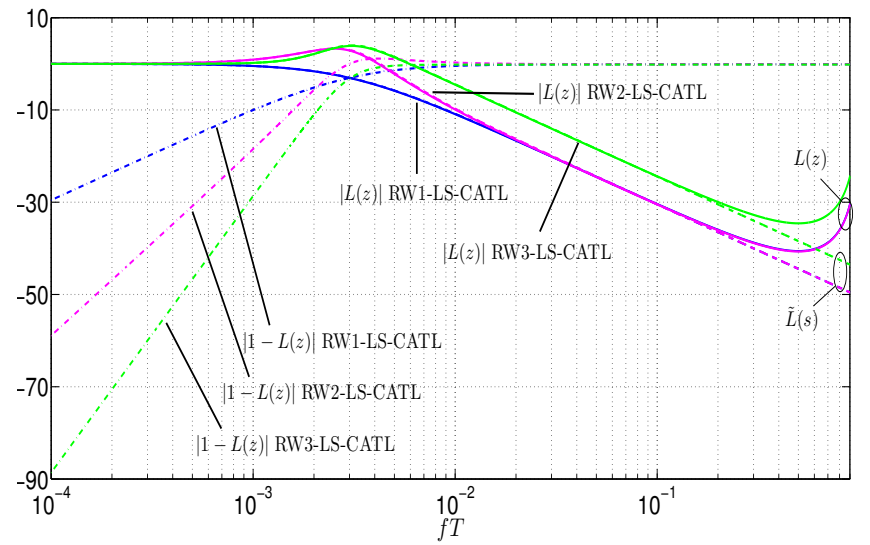

Fig. 3: Magnitude-frequency diagram of $\mathcal{L}\left(e^{j 2 \pi f T}\right), 1-$ $\mathcal{L}\left(e^{j 2 \pi f T}\right), \tilde{\mathcal{L}}\left(e^{j 2 \pi f T}\right)$ (in $\mathrm{dB}$ ) for RW $r$-LS-CATL, loop parameters are optimized for $f_{n} T=3 \cdot 10^{-3}$

The noise variance is calculated by the average loop noise PSD as

$$
\sigma_{\epsilon \mathrm{W}}^{2}=\sigma_{\mathbf{L S}}^{2} \cdot T \int_{-\frac{1}{2 T}}^{+\frac{1}{2 T}}\left|\mathcal{L}\left(e^{j 2 \pi f T}\right)\right|^{2} d f .
$$

1) New global optimization: The optimization of the LSCATL leads to the optimum parameters presented in Table II (second part). Here, we should point out that for the RW3LSCATL, we use a method different from that in [24], where we used a constraint to enhance the linear approximation of 
TABLE II: Asymptotic expressions (first part) and global optimization (second part) for the RW $r$-LS-CATL under the assumption $f_{d} T \leq f_{n} T \ll 1$

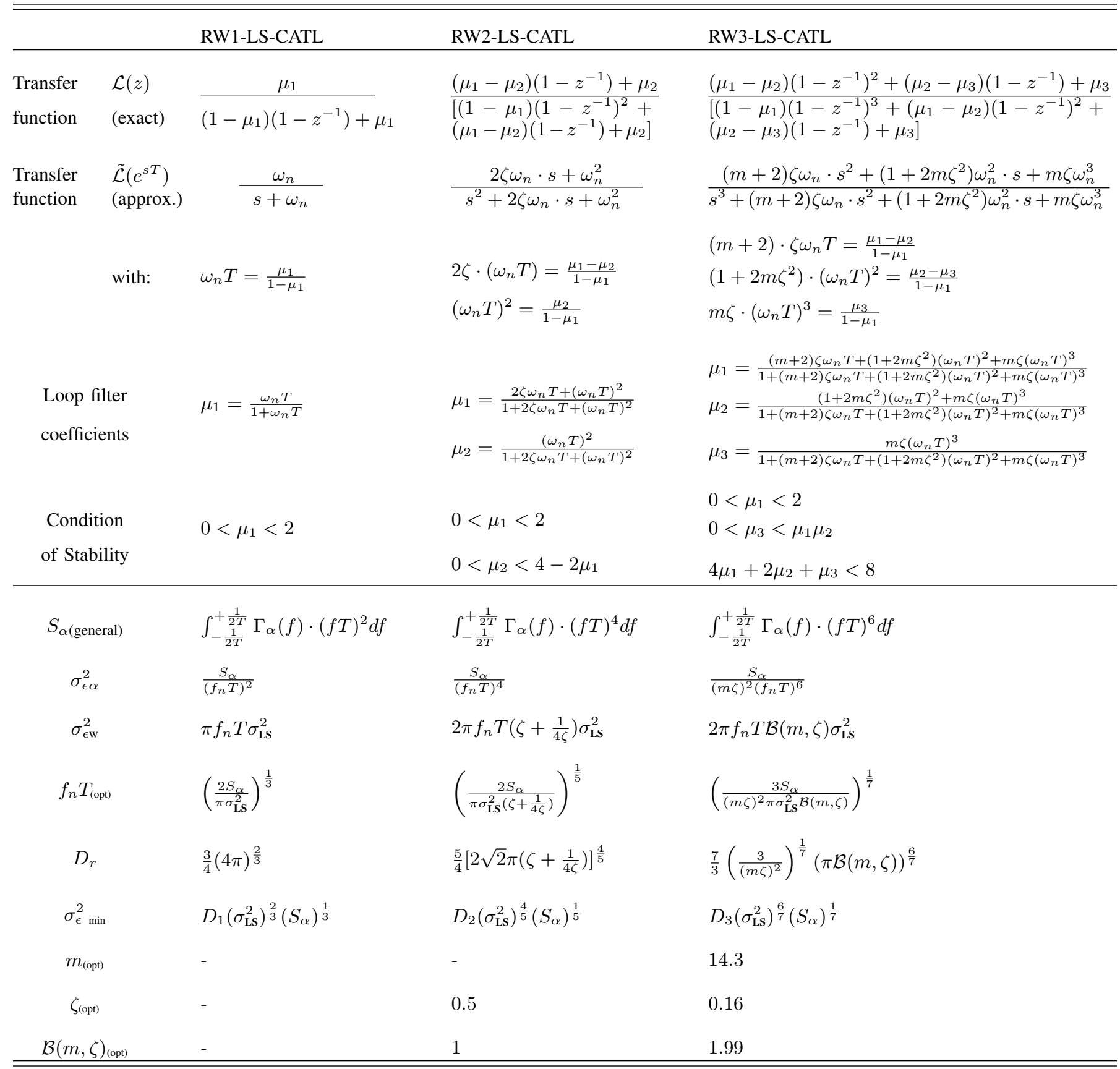

$\left|1-\tilde{\mathcal{L}}\left(e^{j 2 \pi f T}\right)\right|$ (that method leads to a sub-optimal result, as explained in [24] for a specific third-order CATL).

First, we present the results for $\sigma_{\epsilon \alpha}^{2}$ and $\sigma_{\epsilon \mathrm{W}}^{2}$ of Table II (second part). These results are obtained with approximations that are detailed hereafter. In $(30), \mathcal{L}$ is first replaced by $\tilde{\mathcal{L}}$, then $\left|1-\tilde{\mathcal{L}}\left(e^{j 2 \pi f T}\right)\right|^{2}$ is approximated by a linear function of $\left(f / f_{n}\right)^{2 r}$ (asymptote) in the low frequency region $\left(f \ll f_{n}\right)$ :

$$
\left|1-\tilde{\mathcal{L}}\left(e^{j 2 \pi f T}\right)\right|^{2} \approx K_{r} \cdot\left(\frac{f}{f_{n}}\right)^{2 r} \quad \text { for } f \ll f_{n},
$$

where $K_{r}$ is a constant that can be computed by

$$
K_{r}=\left|\lim _{s \rightarrow 0}\left(\frac{\omega_{n}}{s}\right)^{r} \cdot\left(1-\tilde{\mathcal{L}}\left(e^{s T}\right)\right)\right|^{2}
$$

that is, $K_{r}=1$ for $r=1$ and 2 , and $K_{3}=1 /(m \zeta)^{2}$. The validity of this linear approximation can be seen by measuring the slope of the high pass filter $\left|1-L\left(e^{j 2 \pi f T}\right)\right|$ in Fig. 3. This first approximation is made in order to simplify the calculation of the integral in $\sigma_{\epsilon \alpha}^{2}$. We define the term $S_{\alpha \text { (general) }}$ in Table II, which can be viewed as the $2 r$ th moments of the Doppler 
TABLE III: Application of the global optimization for Jakes and 3D Doppler spectra

\begin{tabular}{|c|c|c|c|}
\hline$S_{\alpha(\text { Jakes })}$ & $\frac{1}{2} \cdot\left(f_{d} T\right)^{2} \cdot \frac{\sigma_{\alpha}^{2}}{L}$ & $\frac{3}{8} \cdot\left(f_{d} T\right)^{4} \cdot \frac{\sigma_{\alpha}^{2}}{L}$ & $\frac{5}{16} \cdot\left(f_{d} T\right)^{6} \cdot \frac{\sigma_{\alpha}^{2}}{L}$ \\
\hline$S_{\alpha(3 \mathrm{D})}$ & $\frac{1}{3} \cdot\left(f_{d} T\right)^{2} \cdot \frac{\sigma_{\alpha}^{2}}{L}$ & $\frac{1}{5} \cdot\left(f_{d} T\right)^{4} \cdot \frac{\sigma_{\alpha}^{2}}{L}$ & $\frac{1}{7} \cdot\left(f_{d} T\right)^{6} \cdot \frac{\sigma_{\alpha}^{2}}{L}$ \\
\hline$f_{n} T_{\text {(Jakes) }}$ & $\left(\frac{1}{\pi L} \frac{\sigma_{\alpha}^{2}}{\sigma_{\mathbf{L S}}^{2}}\right)^{\frac{1}{3}}\left(f_{d} T\right)^{\frac{2}{3}}$ & $\left(\frac{3}{4 \pi L} \frac{\sigma_{\alpha}^{2}}{\sigma_{\mathbf{L S}}^{2}}\right)^{\frac{1}{5}}\left(f_{d} T\right)^{\frac{4}{5}}$ & $0.6 \cdot\left(\frac{1}{L} \frac{\sigma_{\alpha}^{2}}{\sigma_{\mathbf{L S}}^{2}}\right)^{\frac{1}{7}}\left(f_{d} T\right)^{\frac{6}{7}}$ \\
\hline$f_{n} T_{(3 \mathrm{D})}$ & $\left(\frac{2}{3 \pi L} \frac{\sigma_{\alpha}^{2}}{\sigma_{\mathbf{L S}}^{2}}\right)^{\frac{1}{3}}\left(f_{d} T\right)^{\frac{2}{3}}$ & $\left(\frac{2}{5 \pi L} \frac{\sigma_{\alpha}^{2}}{\sigma_{\mathbf{L S}}^{2}}\right)^{\frac{1}{5}}\left(f_{d} T\right)^{\frac{4}{5}}$ & $0.54 \cdot\left(\frac{1}{L} \frac{\sigma_{\alpha}^{2}}{\sigma_{\mathbf{L S}}^{2}}\right)^{\frac{1}{7}}\left(f_{d} T\right)^{\frac{6}{7}}$ \\
\hline$\sigma_{\epsilon \min (\text { Jakes) }}^{2}$ & $3.2 \cdot\left(f_{d} T \sigma_{\mathbf{L S}}^{2}\right)^{\frac{2}{3}}\left(\frac{\sigma_{\alpha}^{2}}{L}\right)^{\frac{1}{3}}$ & $5.9 \cdot\left(f_{d} T \sigma_{\mathbf{L S}}^{2}\right)^{\frac{4}{5}}\left(\frac{\sigma_{\alpha}^{2}}{L}\right)^{\frac{1}{5}}$ & $8.7 \cdot\left(f_{d} T \sigma_{\mathbf{L S}}^{2}\right)^{\frac{6}{7}}\left(\frac{\sigma_{\alpha}^{2}}{L}\right)^{\frac{1}{7}}$ \\
\hline
\end{tabular}

TABLE IV: Optimization under constraint (36) for RWr-LS-CATL

\begin{tabular}{clll}
\hline \hline & RW1-LS-CATL & RW2-LS-CATL & RW3-LS-CATL \\
\hline$m_{\text {(sub-opt) }}$ & - & $4 \zeta^{2}-1=0$ & $m^{2}\left(4 \zeta^{2}-1\right)+4=0$ \\
$\zeta_{\text {(sub-opt) }}$ & - & - & 3.19 \\
$\mathcal{B}(m, \zeta)_{\text {(opt) }}$ & - & $\equiv$ & 0.39 \\
$f_{n} T_{\text {(Jakes) }}$ & $\equiv$ & $\equiv$ & $0.73 \cdot\left(\frac{1}{L} \frac{\sigma_{\alpha}^{2}}{\sigma_{\mathbf{L S}}^{2}}\right)^{\frac{1}{7}}\left(f_{d} T\right)^{\frac{6}{7}}$ \\
$f_{n} T_{\text {(3D) }}$ & $\equiv$ & $\equiv$ & $0.65 \cdot\left(\frac{1}{L} \frac{\sigma_{\alpha}^{2}}{\sigma_{\mathbf{L S}}^{2}}\right)^{\frac{1}{7}}\left(f_{d} T\right)^{\frac{6}{7}}$ \\
$\sigma_{\epsilon \text { min (Jakes) }}^{2}$ & $\equiv$ & $\equiv$ & $9.3 \cdot\left(f_{d} T \sigma_{\mathbf{L S}}^{2}\right)^{\frac{6}{7}}\left(\frac{\sigma_{\alpha}^{2}}{L}\right)^{\frac{1}{7}}$ \\
$\sigma_{\epsilon \text { min (3D) }}^{2}$ & $\equiv$ & $\equiv$ & $8.31 \cdot\left(f_{d} T \sigma_{\mathbf{L S}}^{2}\right)^{\frac{6}{7}}\left(\frac{\sigma_{\alpha}^{2}}{L}\right)^{\frac{1}{7}}$
\end{tabular}

N.B.: "E" stands for the identical value of the corresponding terms in Table II and III.

spectrum [27]:

$$
S_{\alpha(\text { general })}=\int_{-\frac{1}{2 T}}^{+\frac{1}{2 T}} \Gamma_{\alpha}(f) \cdot(f T)^{2 r} d f .
$$

Now, $\sigma_{\epsilon \alpha}^{2}$ can be written in closed-form as a function of $S_{\alpha(\text { general })}$ :

$$
\sigma_{\epsilon \alpha}^{2}=\frac{S_{\alpha(\text { general })}}{\left(f_{n} T\right)^{2 r}} \cdot K_{r}
$$

This allows obtaining a general expression for $\sigma_{\epsilon \alpha}^{2}$ for any (narrow-band) channel spectrum $\Gamma_{\alpha}(f)$. The $S_{\alpha \text { (general) }}$ are then specified for the flat (3D) and the Jakes Doppler spectrum in Table III.

Then, a second approximation is used to simplify the calculation of $\sigma_{\epsilon \mathrm{W}}^{2}$. It consists in neglecting the higher order terms of $\omega_{n} T$ when calculate the equivalent noise bandwidth of the estimator, since $f_{n} T \ll 1$. Then we can obtain the simplified noise bandwidth, and then $\sigma_{\epsilon \mathrm{W}}^{2}$ is proportional to $2 \pi f_{n} T \sigma_{L S}^{2}$, as shown in Table II. The coefficient of proportionality is fixed to $\mathcal{B}=\frac{1}{2}$ for RW1, and is $\mathcal{B}(\zeta)=\zeta+\frac{1}{4 \zeta}$ for RW2 [5], with then a dependency on $\zeta$. Note that for $\mathrm{RW} 3, \mathcal{B}(m, \zeta)$ is a coefficient which depends on $m$ and $\zeta$ [24]:

$$
\mathcal{B}(m, \zeta)=\frac{2 m^{3} \zeta^{4}+12 m^{2} \zeta^{4}+8 m \zeta^{4}+6 m \zeta^{2}+4 \zeta^{2}+1}{4 m^{2} \zeta^{3}+8 m \zeta^{3}+4 \zeta} .
$$

Second, we present the global optimization results for the $r$ parameters. The derivations are detailed in the Appendix. The minimization of $\sigma_{\epsilon}^{2}$ consists in setting to zero the partial derivative w.r.t. each of the $r$ parameters, i.e., $f_{n}, \zeta$, and $m$ for $r=3$. By solving these equations, we first find the 
optimal $f_{n}$ of the loop as a function of the SNR, $S_{\alpha}, \zeta$, and $m$ according to the order. That optimal $f_{n}$ is given in Table II (second part). Then the optimal $\zeta$ and $m$ (according to the order) can be found directly, without any dependence on the channel parameters (the SNR and $f_{d} T, S_{\alpha}$ ). Consequently, only one parameter, i.e., $f_{n}$, has to be tuned regardless of the loop order. The remaining $r-1$ parameters can be fixed to their optimal values, as given in the table. This remarkable result is illustrated by Fig. 2 for the Jakes or 3D Doppler models and for two values of the SNR. The red points correspond to the global optimization obtained by our theoretical formulae of Table II. For the third order loop, it can be seen from Fig. 2 c) to $\mathrm{f}$ ) that the optimum values of $m$ and $\zeta$ are quite independent of the channel parameters and are fixed to $\zeta_{\text {(opt) }}=0.16$ and $m_{\text {(opt) }}=14.3$, according to the theory (see the Appendix and Table II). In the same way for the second order loop, the optimization of $\zeta$ leads to the same value $\zeta_{\text {(opt) }}=0.5$, regardless of the channel parameters (see Fig. 2(b)). Finally, for the first order loop, only $f_{n}$ can be optimized. We can check in Fig. 2(a) that the minimum point's position varies as a function of SNR, $f_{d} T$, and $S_{\alpha}$ in accordance with the theory. More comparisons between the MSE obtained with given parameters and the exact minimum MSE (obtained by means of a numerical search of the actual best parameters) were performed in our previous studies [24](Fig. 3, Fig. 4) for order 3 with Jakes's spectrum, and in [5](Fig. 3, Fig. 4, Fig.5) for orders 1 and 2 with Jakes's spectrum.

2) Optimization with constraint (review): We recall that for RW3-LS-CATL, it is also possible to find a sub-optimal result by using a constraint:

$$
\left|1-\tilde{\mathcal{L}}\left(e^{j 2 \pi f T}\right)\right|^{2}=K_{r} \cdot\left(\frac{f}{f_{n}}\right)^{2 r} \quad \text { for } f=f_{n},
$$

which means that we require that the low-pass transfer function line passes the asymptote at the point $f=f_{n}$. This has been intuitively shown in [24, Fig.2] for RW3-CATL. With constraints, we can exploit the method of Lagrange to optimize the estimator, which yields $m_{\text {(sub-opt) }}=3.19$ and $\zeta_{\text {(sub-opt) }}=0.39$. The constraints and the sub-optimal results with constraint are given in Table IV, and are illustrated in Fig. 2 (c) to (f). We notice that for the third order loop, this sub-optimal point (black point) is not near the optimal point in the $m-\zeta$ plane, but the MSE values are very close since they are both in the min-value valley [24, fig. 3]. Note also that this constraint can be applied to the second order loop and it yields the same result as with the global optimization, that is $\zeta=0.5$.

\section{E. Equivalence of $R W r-L S$-CATL channel estimation for flat (3D) and Jakes's Doppler spectrum}

For the afore-mentioned Rayleigh-Jakes and the flat (3D) models, the moment $S_{\alpha}$ can be found in closed form:

$$
S_{\alpha}=S \cdot\left(f_{d} T\right)^{2 r} \cdot \frac{\sigma_{\alpha}^{2}}{L},
$$

where $S$ is a constant depending on the chosen spectrum and loop order. Then the closed form minimum AMSE of the RWr-LS-CATL can be obtained: it is proportional to the $\frac{2 r}{2 r+1}$ th power of the path loop noise variance $\sigma_{\mathrm{LS}}^{2}$, and is also proportional to the $\frac{2 r}{2 r+1}$ th power of $f_{d} T$ (all detailed results are given in Table III).

In the two special cases of flat and Jakes's Doppler spectrum, we can find the equivalent maximum Doppler frequencies for a target performance of the RWr-LS-CATL. For example, let us define the maximum Doppler frequency of these two channel models as $f_{d(\mathrm{Jakes})}$ and $f_{d(3 \mathrm{D})}$ respectively (we assume that these frequencies are less than $\frac{1}{T s}$ ). In order that $\sigma_{\epsilon \text { (Jakes) }}^{2}=\sigma_{\epsilon(3 \mathrm{D})}^{2}$, their dynamic parts should be identical:

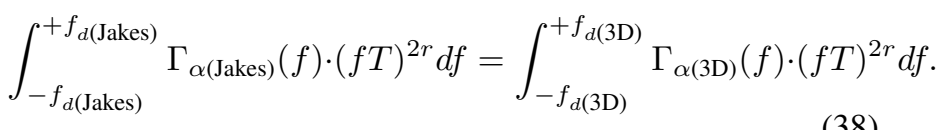

We can find the equivalent Doppler frequency of the Jakes and the 3D model by letting $S_{\alpha \text { (Jakes) }}=S_{\alpha(3 \mathrm{D})}$ for RW $r$-LS-CATL, yielding (from first and second rows of Table III):

$$
\begin{array}{ll}
f_{d(3 \mathrm{D})} / f_{d(\text { Jakes })}=\sqrt{\frac{3}{2}} & \text { for } r=1, \\
f_{d(3 \mathrm{D})} / f_{d(\text { Jakes })}=\sqrt[4]{\frac{15}{8}} & \text { for } r=2, \\
f_{d(3 \mathrm{D})} / f_{d(\text { Jakes })}=\sqrt[6]{\frac{35}{16}} & \text { for } r=3 .
\end{array}
$$

In other words, to retain the same estimation performance, the range of $f_{d(3 \mathrm{D})}$ should be $\sqrt{3 / 2}, \sqrt[4]{15 / 8}, \sqrt[6]{35 / 16}$ times larger than $f_{d \text { (Jakes) }}$. The result for the first order loop, based on the second-order moment, corroborates that obtained in [27].

\section{F. Connection between RW-LS-CATL and RW-KF (optimized in Jakes's case)}

In the single-path single-carrier scenario (Jakes's Doppler spectrum), the RWr-KF based estimator has been proved to be equivalent to the same order RWr-CATL with fixed given parameters [13], [19], [24]. However, in the multi-path multicarrier scenario, the equivalence between the RWr-LS-CATL and the RWr-KF is not aways available: the former processes the paths separately, and optimized by the average path power, whereas the latter processes the multi-path channels jointly. However, a link between the RW $r$-LS-CATL and a per-path processing $\mathrm{RW} r-\mathrm{KF}$ [28] can be found.

A per-path $\mathrm{RW} r-\mathrm{KF}$ is a multi-path channel estimator that processes each path separately with a single-path $\mathrm{RW} r-\mathrm{KF}$. Each single-path KF is optimized by the channel state noise variance. It has been shown that the per-path processing KF can have almost the same performance as the joint multipath KF for the AR1 model [29], and this approximation has been analytically proved for RW models [28]. The per-path and joint multipath KF are identical when the discrete-time equivalent channel is uncorrelated, i.e., $\mathbf{F}_{\mathbf{p}}^{H} \mathbf{F}_{\mathbf{p}}=N_{p} \mathbf{I}_{L}$, or, equivalently, the multipath delays are multiples of the sample time, i.e., $\tau^{(l)}$ are integers ( [28], appendix A).

The global MSE of the optimized per-path RWr-KF is ( [28])

$$
\sigma_{\epsilon \min }^{2}=C_{r} \cdot\left(f_{d} T\right)^{\frac{2 r}{2 r+1}} \cdot\left(\sigma_{\mathrm{w}}^{2}\right)^{\frac{2 r}{2 r+1}} \cdot \beta_{r},
$$

where $C_{r}$ is a constant related to the KF order: $C_{1}=$ $\frac{3}{2} \pi^{\frac{2}{3}}, C_{2}=\frac{15}{8}(\sqrt{2} \pi)^{\frac{4}{5}}, C_{3}=\frac{35}{16}\left(\frac{16}{9} \pi\right)^{\frac{6}{7}}$. The coefficients $\beta_{r}$ 
( $r=1,2,3)$ are noise factors that depend on the channel PDP, and are defined by

$$
\beta_{r}=\frac{1}{L} \sum_{l=1}^{L}\left\{\left[\left(\mathbf{F}_{\mathbf{p}}^{H} \mathbf{F}_{\mathbf{p}}\right)^{-1}\right]_{l, l}^{\frac{2 r}{2 r+1}} \cdot\left(\sigma_{\alpha^{(l)}}^{2}\right)^{\frac{1}{2 r+1}}\right\} .
$$

This formula shows that the asymptotic performance of $\mathrm{RW} r$ $\mathrm{KF}$ varies the same as the RW $r$-LS-CATL, as a function of $\left(f_{d} T\right)^{\frac{2 r}{2 r+1}}$ and $\left(\sigma_{\mathrm{w}}^{2}\right)^{\frac{2 r}{2 r+1}}$. However, the coefficient $\beta_{r}$ depends on the channel PDP. If all the paths are supposed to have the same attenuation, then $\beta_{r}$ can be simplified:

$$
\beta_{r}=\left(\frac{\sigma_{\alpha}^{2}}{L}\right)^{\frac{1}{2 r+1}} \cdot \frac{1}{L} \sum_{l=1}^{L}\left[\left(\mathbf{F}_{\mathbf{p}}^{H} \mathbf{F}_{\mathbf{p}}\right)^{-1}\right]_{l, l}^{\frac{2 r}{2 r+1}}
$$

By using (25) and by letting $\frac{2 r}{2 r+1}$ approach 1 ,

$$
\left(\sigma_{\mathbf{L S}}^{2}\right)^{\frac{2 r}{2 r+1}} \approx \frac{1}{L} \sum_{l=1}^{L}\left\{\sigma_{\mathrm{w}}^{2}\left[\left(\mathbf{F}_{\mathbf{p}}^{H} \mathbf{F}_{\mathbf{p}}\right)^{-1}\right]_{l, l}\right\}^{\frac{2 r}{2 r+1}}
$$

we can rewrite (42) as

$$
\sigma_{\epsilon \min }^{2} \approx C_{r} \cdot\left(f_{d} T\right)^{\frac{2 r}{2 r+1}} \cdot\left(\sigma_{\mathrm{LS}}^{2}\right)^{\frac{2 r}{2 r+1}} \cdot\left(\frac{\sigma_{\alpha}^{2}}{L}\right)^{\frac{1}{2 r+1}} .
$$

The relation between $\mathrm{RW} r$-KF and RW $r$-LS-CATL can thus be found by comparing with $\sigma_{\epsilon}^{2} \min$ (Jakes) in Table III: similar to the single-path single-carrier scenario, for RW1, they have the same asymptotic performance; for RW2, if we choose $\zeta=\sqrt{2} / 2$ for LS-CATL, we can reach the same asymptotic performance as the KF optimized in [19]. For RW3, an LSCATL with $m=2$ and $\zeta=0.5$ can reach the same AMSE as the KF optimized in [13]. We can see clearly in Fig. 2(c, d) that this point $m=2$ and $\zeta=0.5$ (green point) is far away from the global minimum point $m=14.3$ and $\zeta=0.16$ but leads to a very close AMSE since it is still in the min-value valley.

To sum up, RWr-LS-CATL and RWr-KF are not strictly equivalent in steady-state mode, but are strongly connected: the RWr-LS-CATL can be viewed as a steady-state mode perpath RWr-KF. When assuming a energy-uniformly-distributed multi-path channel, they can thus achieve very close asymptotic performances.

\section{G. Algorithmic complexity}

One can compute the number of operations of complex multiplication in the iteration equations of the tracking loop (15) (20), with (15) defined by (21). Note that the matrices $\mathbf{G}_{(k)}$ can be pre-calculated (assuming a fixed pilot pattern), so their contribution to the algorithmic complexity is negligible when the number of transmitted OFDM symbols is large. Thus, in fact, the complex multiplication in (15) is counted only in (21). Then we have, for (15), $N_{p} \times L$ multiplications; for (18), $r \times L$ multiplications (between a real and a complex number). The algorithmic complexity of RW $r$-LS-CATL is $O\left(N_{p}\right)$.

For the joint multipath $\mathrm{RW} r-\mathrm{KF}$, the number of complex multiplications is $N_{p}^{3}+(r+1) L N_{p}^{2}+\left(r^{2} L^{2}+r L^{2}+r L+\right.$ $L+1) N_{p}$ [28]. The complexity of RW $r$-KF is $O\left(N_{p}^{3}\right)$, which is much higher than that of RWr-LS-CATL.
TABLE V: Complex multiplications in RWr-LS-CATL (per iteration)

\begin{tabular}{lcc}
\hline \hline Operation & Equation & Multiplications \\
\hline $\mathbf{G}_{(k)} \mathbf{y}_{\mathbf{p}(k)}$ & $(21)$ & $N_{p} L$ \\
$\begin{array}{l}\mu_{1} \boldsymbol{v}_{\boldsymbol{\epsilon}(k)}+\mu_{2} \boldsymbol{v}_{\text {Lag1 }(k)}+ \\
\mu_{3} \boldsymbol{v}_{\text {Lag2 }(k-1)}\end{array}$ & $(18)$ & $\frac{1}{2} r L$ \\
\hline \hline
\end{tabular}

\section{Simulation}

In the simulations, we used a 4QAM-OFDM (by default) system with $N=128$ sub-carriers to validate the proposed approximate method and the analytic results. The OFDM system has $N_{g}=16$ samples of $\mathrm{CP}$, and the system bandwidth is $1 / T_{s}=2 \mathrm{MHz}$. The channel delays $\tau^{(l)}$ are assumed perfectly known, but can be estimated with algorithms such as the ESPRIT [35]. The impact of an estimation error has been investigated in [36] which showed the good robustness of the CATL.

First, the evolution of the factor $\lambda$ as a function of the number of pilot sub-carriers will be illustrated. We recall that the noise amplification factor (compared to the single-path single-carrier performance) would be equal to 1 (minimum value) when the path delays of the channel are multiples of the sampling time $T_{s}=500 \mathrm{~ns}$ in our case. Two types of channels with different power delay profiles (given in Table VI) were used, the GSM [37] and the WiMAX mobile (Vehicular A) 6-tap outdoor channel models. For the rest of the simulations, only the GSM channel model was used and the number of pilot sub-carriers for each OFDM symbol was fixed at $N_{p}=16$.

For a given channel PDP, $\lambda$ can be calculated by (27): it decides the level of the loop noise. Note that the value of $\lambda$ depends on both the number of pilot sub-carriers and the channel PDP. Table VII gives the values of $\lambda$ in two types of channels. We find that $\lambda$ decreases as the number of pilot subcarriers increases. The WiMAX mobile channel corresponds to a lower value of $\lambda$ than does the GSM channel. According to (26), the value of $\lambda$ is proportional to the static error variance. From Fig. 4, we find that the MSE values (which vary as a function of $N_{p}$ ) in a GSM channel are always higher than those obtained in a WiMAX channel, since the former channel PDP corresponds to a larger $\lambda$. The theoretical values are then validated by the simulations for RW1-, RW2- and RW3-LSCATL.

The optimal natural (cut-off) frequencies with respect to $N_{p}$ and SNR, for the two typical channels, are also summarized in Tables VIII and IX, respectively. These two tables show that the optimal natural (cut-off) frequencies of $\mathrm{RW} r$-LSCATL increase as the number of pilot sub-carriers increases, and also as the SNR. The higher the loop order, the slower the augmentation of its optimal natural frequencies (with respect to $N_{p}$ or SNR). Besides, the optimal natural (cutoff) frequencies for a GSM channel are smaller than those for WiMAX, because the loop noise level is relatively higher in the GSM case, and the optimal natural (cut-off) frequencies 


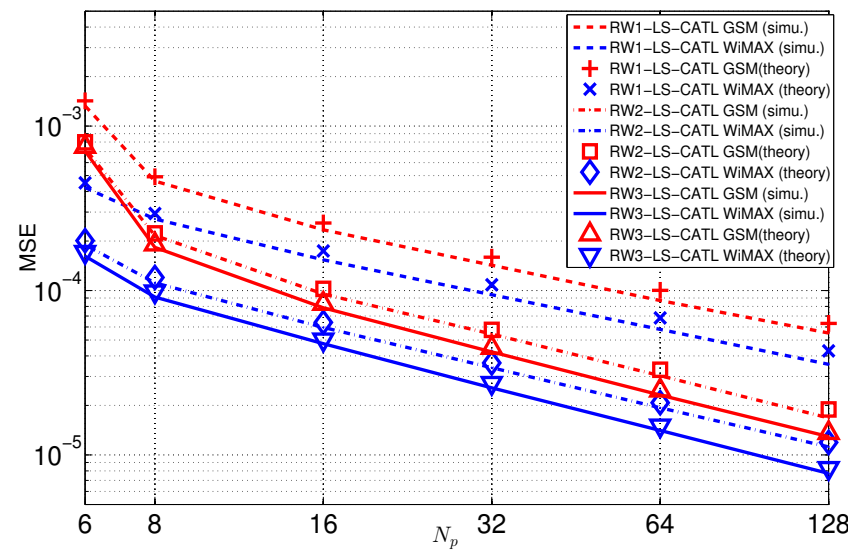

Fig. 4: AMSE versus $N_{p}$ (number of pilot sub-carriers) with $f_{d} T=10^{-3}, \mathrm{SNR}=20 \mathrm{~dB}$

are decreasing functions of the loop noise variance.

Fig. 5 shows the simulated AMSE (mean value per path) of the $\mathrm{AR} 1_{C M}$-KF and the RW-LS-CATLs, the theoretical AMSE of the RW-LS-CATLs, and the BCRB as functions of the SNR for $f_{d} T=10^{-3}$. Fig. 6 presents the MSE evolution as a function of $f_{d} T$. We can see clearly that the RW-LS-CATLs outperforms the $\mathrm{AR} 1_{C M}-\mathrm{KF}$. Moreover, the MSEs obtained by the simulation approximately coincide with the theoretical values. Note that we have made approximations for the analytical expressions of the AMSE employing the assumption of a low $f_{d} T$ and a low $f_{n} T$, such as $f_{d} T \leq f_{n} T \ll 1$; these approximations becomes poorer when $f_{d} T$ increases, as shown in Fig. 6, and when SNR increases, as shown in Fig. 5. Also we can note that in these cases, the error for first order model is getting larger compared to the second and third order models. For $r=1,2,3$, the AMSE are respectively proportional to the $2 / 3,4 / 5,6 / 7$ powers of the $f_{d} T$, and are inversely proportional to the $2 / 3,4 / 5,6 / 7$ powers of the $S N R$, as argued in the previous section. In addition, we show the simulation results of a numerically optimized RW $r$-KF in Fig. 5, to show that the simplified RW $r$-LS-CATL can reach almost the same asymptotic performance as the RW $r$-KF.

The equivalence of the RWr-LS-CATL channel estimation in the flat and the Jakes Doppler spectrum was verified. First we fixed the Doppler frequency $f_{d} T$ at $10^{-3}$ to simulate the Jakes spectrum channel by RW $r$-LS-CATL $(r=1,2$ and 3). Then, we simulated the flat (3D) spectrum channel by using $f_{d}^{\prime} T / f_{d} T=\sqrt{3 / 2}, \sqrt[4]{15 / 8}, \sqrt[6]{35 / 16}$ for RW1, 2, 3 respectively. The simulation results are given in Fig. 7. These two groups of curves are very close to each other, which confirms our previous analysis in section III-E. We can conclude that for a given $f_{d} T$, the channel estimation with flat spectrum (3D) channel has a better performance than that with Jakes's spectrum channel.

In Fig. 8, the BER performances of the RW-LS-CATL, the $\mathrm{AR} 1_{C M}-\mathrm{KF}$, and the conventional non-parametric channel estimators are compared. For symbol decision, the estimators are completed by a Zero-forcing (ZF) equalizer in the frequency domain, i.e., $\hat{\mathbf{x}}_{(k)}=\hat{\mathbf{H}}_{(k)}^{-1} \mathbf{y}_{(k)}$ with $\hat{\mathbf{H}}_{(k)}=\operatorname{diag}\left\{\mathbf{F} \hat{\boldsymbol{\alpha}}_{(k)}\right\}$. The $\mathrm{AR} 1_{C M}-\mathrm{KF}$ is a Kalman estimator based on the first-order AR model. Note that the first two algorithms are based on the parametric channel model and estimate the complex amplitude of the channel, whereas the non-parametric estimators estimate the frequency-domain channel matrix without presuming any knowledge of the channel PDP. The conventional frequencydomain algorithms are presented in [2]-[4], and are based on the observation models: $\mathbf{y}_{\mathbf{p}(k)}=\operatorname{diag}\left\{\mathbf{x}_{\mathbf{p}(k)}\right\} \mathbf{h}_{\mathbf{p}(k)}+\mathbf{w}_{\mathbf{p}(k)}$, without exploiting the knowledge of the channel PDP. The first algorithm, LS(FD)-LPI, exploits the LS method to estimate the diagonal elements of the channel matrix on the pilot sub-carriers: $\hat{\mathbf{h}}_{\mathbf{p}(k)}=\operatorname{diag}\left\{\mathbf{x}_{\mathbf{p}(k)}\right\}^{-1} \mathbf{y}_{\mathbf{p}(k)}$. Then a low-pass interpolation (LPI) is carried out to obtain the full channel matrix $\hat{\mathbf{H}}_{(k)}$ (a diagonal matrix containing the frequency response on its main diagonal), by using the Matlab $b^{\mathrm{TM}}$ function "interp". For the second algorithm, AR1-KF(FD)-LPI [6], after the LS estimation of the pilot channel matrix, as in the first algorithm, a time-domain filtering is processed by $\mathrm{AR} 1_{C M^{-}}$ $\mathrm{KF}$ to refine the pilot channel matrix. Then the same LPI procedure is executed. It is noteworthy that the channel PDP is not exploited in either of the two conventional methods, in contrast with the parametric channel estimations. It can be seen that, with 8 pilots, the parametric methods largely outperform the conventional non-parametric algorithms. Their performances are brought closer as the number of pilot subcarriers increases. Among the parametric channel estimators, the first-order algorithms (AR1 $C M-\mathrm{KF}$ and RW1-LS-CATL) have similar performances. The RW2- and RW3-LS-CATL outperform the first-order ones, with the BER lines almost reaching the performance with perfect Channel State Information (CSI).

Fig. 9 shows for $f_{d} T=10^{-3}$ and $N_{p}=8$ the Bit Error Rate (BER) performances for the proposed RW-LS-CATLs with and without coding, using a zero-forcing equalizer and a 64-QAM modulation. Also shown is the performance for the $\mathrm{AR} 1_{C M}-\mathrm{KF}$. For reference, we have added the BER for the case of perfect CSI. Without coding, we can observe that the $\mathrm{AR} 1_{C M}$-KF curve and the RW1-LS-CATL curve are very

TABLE VI: Power delay profile of the simulation channels

\begin{tabular}{|c|c|c|c|c|c|c|c|}
\hline Channel & Path & 1 & 2 & 3 & 4 & 5 & 6 \\
\hline & $\tau^{(l)}$ & 0 & 0.4 & 1 & 3.2 & 4.6 & 10 \\
\hline GSM & $\sigma_{\alpha}^{2}(l)(\mathrm{dB})$ & -7.219 & -4.219 & -6.219 & -10.219 & -12.219 & -14.219 \\
\hline WiMAX & $\tau^{(l)}$ & 0 & 0.62 & 1.42 & 2.18 & 3.46 & 5.02 \\
\hline mobile & $\sigma_{\alpha}^{2}(l)(\mathrm{dB})$ & -3.1425 & -4.1425 & -12.1425 & -13.1425 & -18.1425 & -23.1425 \\
\hline
\end{tabular}


TABLE VII: Evolution of $\lambda$ with respect to $N_{p}$

\begin{tabular}{crrrrrr}
\hline \hline$N_{p}$ & 6 & 8 & 16 & 32 & 64 & 128 \\
\hline$\lambda(\mathrm{GSM})$ & 13.271 & 3.703 & 2.804 & 2.736 & 2.725 & 2.722 \\
\hline$\lambda($ WiMAX mobile) & 2.445 & 1.711 & 1.559 & 1.535 & 1.529 & 1.528 \\
\hline \hline
\end{tabular}

TABLE VIII: Optimal normalized natural frequencies $f_{n} / f_{d}$ versus $N_{p}(\mathrm{SNR}=20 \mathrm{~dB})$

\begin{tabular}{crrrrrrr}
\hline \hline & & & & & & & \\
& $N_{p}$ & & 6 & 8 & 16 & 32 & 128 \\
\hline \multirow{2}{*}{ GSM } & RW1 & 13.25 & 22.55 & 31.16 & 39.59 & 49.95 & 62.95 \\
& RW2 & 4.45 & 6.12 & 7.43 & 8.58 & 9.87 & 11.34 \\
& RW3 & 2.61 & 3.27 & 3.76 & 4.17 & 4.60 & 5.08 \\
\hline \multirow{2}{*}{ WiMAX mobile } & RW1 & 23.52 & 29.16 & 37.90 & 48.00 & 60.55 & 76.31 \\
& RW2 & 6.28 & 7.14 & 8.36 & 9.63 & 11.07 & 12.72 \\
& RW3 & 3.33 & 3.66 & 4.09 & 4.53 & 5.00 & 5.52 \\
\hline \hline
\end{tabular}

TABLE IX: Optimal normalized natural frequencies $f_{n} / f_{d}$ versus SNR $\left(N_{p}=16\right)$

\begin{tabular}{lrrrrrrrrrr}
\hline \hline \multicolumn{2}{c}{ SNR (dB) } & 0 & 5 & 10 & 15 & 20 & 25 & 30 & 35 & 40 \\
\hline \multirow{2}{*}{ GSM } & RW1 & 6.7 & 9.9 & 14.5 & 21.2 & 31.2 & 45.7 & 67.1 & 98.5 & 145 \\
& RW2 & 3.0 & 3.7 & 4.7 & 5.9 & 7.4 & 9.4 & 11.8 & 14.8 & 18.7 \\
& RW3 & 1.9 & 2.3 & 2.7 & 3.2 & 3.8 & 4.4 & 5.2 & 6.2 & 7.3 \\
\hline \multirow{2}{*}{ WiMAX } & RW1 & 8.2 & 12.0 & 17.6 & 25.8 & 37.9 & 55.6 & 81.7 & 120 & 176 \\
mobile & RW2 & 3.3 & 4.2 & 5.3 & 6.6 & 8.4 & 10.5 & 13.3 & 16.7 & 21 \\
& RW3 & 2.1 & 2.5 & 2.9 & 3.5 & 4.1 & 4.8 & 5.7 & 6.8 & 7.9 \\
\hline \hline
\end{tabular}

close. Furthermore, the RW2-LS-CATL curve and the RW3LS-CATL curve nearly coincide. At a BER target of $10^{-3}$, there is an SNR loss of about $2 \mathrm{~dB}$ between the RW2-LSCATL and the RW1-LS-CATL. Then, we use a Non-Recursive NonSystematic Convolutional (NRNSC) code $[5,7]_{8}$. This time, the RW1-LS-CATL curve is far from the $\mathrm{AR} 1_{C M}$-KF and closer to the RW2-LS-CATL curve. At a BER target of $10^{-5}$, there is an SNR loss of about $2.5 \mathrm{~dB}$ between the RW1-LS-CATL and the AR1 $1_{C M}$-KF curves, and only $0.5 \mathrm{~dB}$ between the RW1-LS-CATL and RW2-LS-CATL curves.

In Fig.10, we show the tracking performance of the RW $r$ LS-CATL for the three orders, $r=1,2,3$. As expected, it is seen that the convergence speed of the algorithm is decreasing with the model order.

\section{CONCLUSiON}

In this study, the tracking loops for channel estimation were thoroughly analysed in the context of OFDM systems under the conditions of slow to moderate fading. The analytic optimization of the first three order loops was carried out globally, in contrast with the treatments in the literature, where the use of a constraint yields sub-optimal coefficients. The loop coefficients are the natural frequency $f_{n}$ for the firstorder case, $f_{n}$ and the damping factor $\zeta$ for the second-order case, and $f_{n}, \zeta$, and the capacitance ratio $m$ for the thirdorder case. One remarkable aspect of this global optimization is that only $f_{n}$ has to be tuned as a function of the transmission parameters, i.e., the channel Doppler spectrum, the power

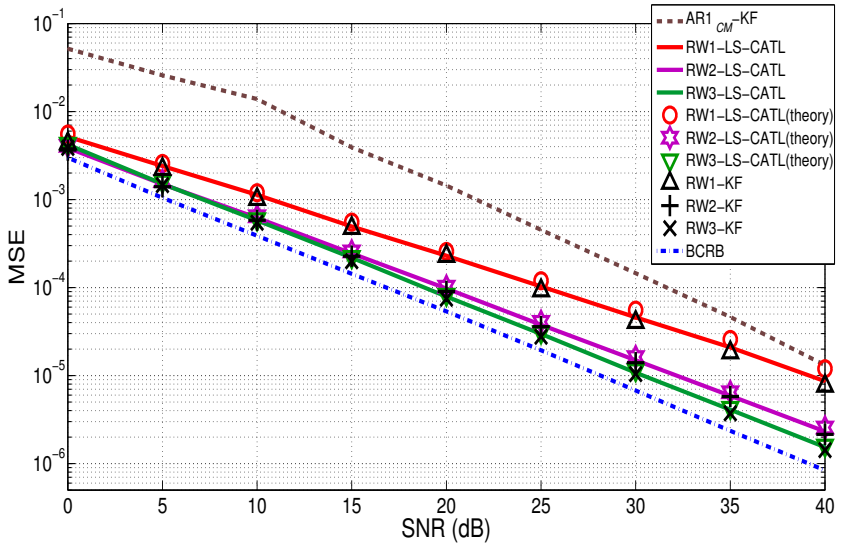

Fig. 5: AMSE versus the SNR with $f_{d} T=10^{-3}, N_{p}=16$, GSM channel

delay profile, and the noise variance. For the second-order case, the optimal value of $\zeta$ is fixed at 0.5 and for the thirdorder case, the optimal values of $\zeta$ and $m$ are fixed at 14.3 and 0.16 , respectively, regardless of the transmission conditions. Moreover, the asymptotic performance is formulated in a new way as a function of the $2 r$ th moment of the Doppler spectrum, where $r$ is the loop order. This way, our results are not limited to the Jakes Doppler spectrum, and can be applied to any Doppler spectrum as long as its $2 r$ th moment is known. In addition, based on this new formulation, we have generalised to the second and third order cases the link between the maxi- 


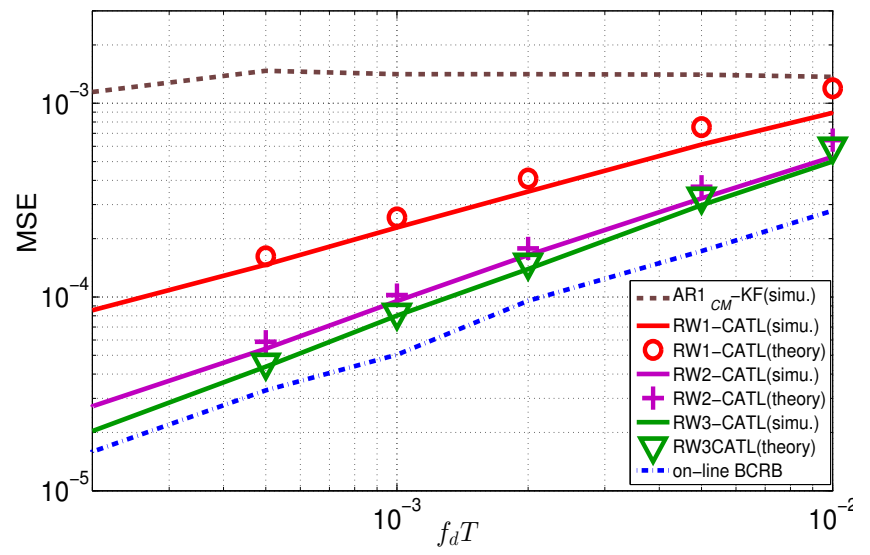

Fig. 6: AMSE versus $f_{d} T$ with $\mathrm{SNR}=20 \mathrm{~dB}, N_{p}=16$, GSM channel

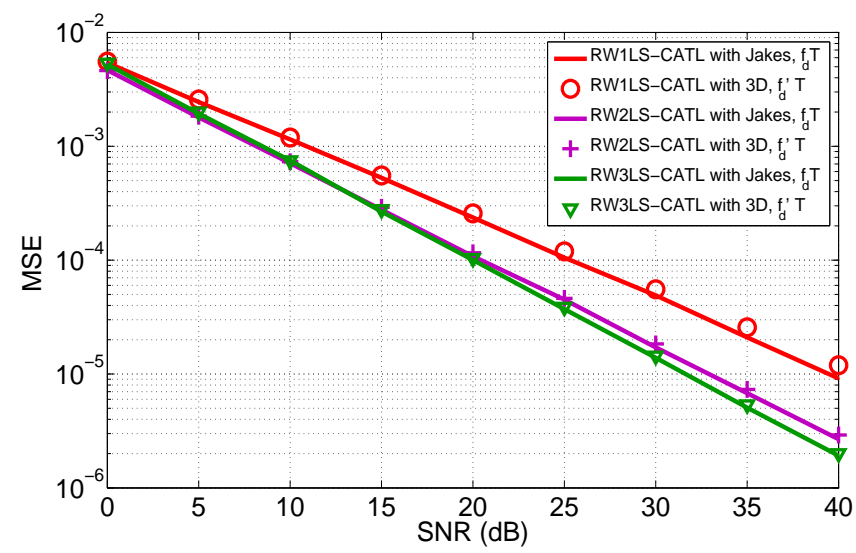

Fig. 7: AMSE versus the SNR with $N_{p}=16$, GSM channel, $f_{d} T=10^{-3}$, and $f_{d}^{\prime} T / f_{d} T=\sqrt{3 / 2}, \sqrt[4]{15 / 8}, \sqrt[6]{35 / 16}$ for $\mathrm{RW} 1,2,3$ respectively

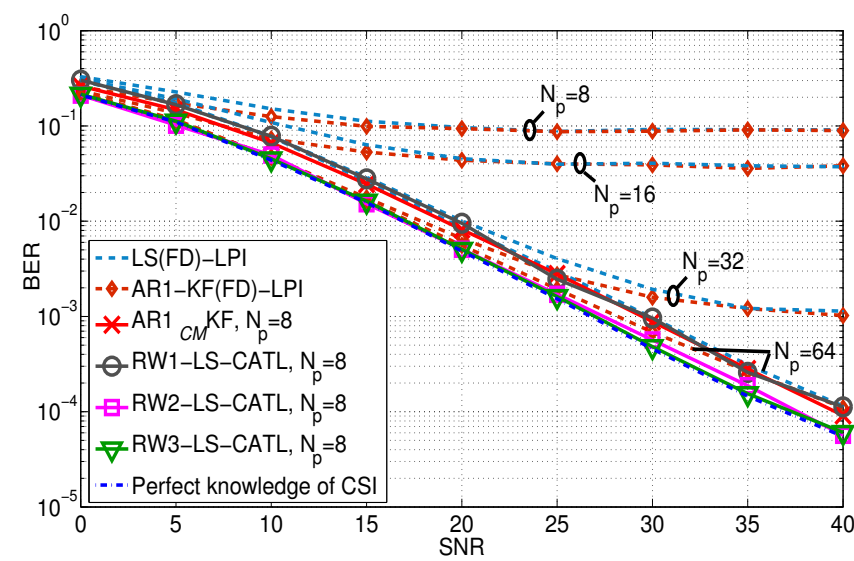

Fig. 8: BER versus SNR with $f_{d} T=10^{-3}$, GSM channel

mum frequency of the Jakes Doppler spectrum $f_{d \text { (Jakes) }}$ and the maximum frequency of the 3D model Doppler spectrum $f_{d(3 \mathrm{D})}$ that give the same asymptotic performance. More precisely, it had been established in the literature that $f_{d(3 \mathrm{D})} / f_{d(\mathrm{Jakes})}=\sqrt{\frac{3}{2}}$ ensures that the second moments of the Doppler spectra are identical. From our formulae, we proved that this leads to the same asymptotic performance for the first order loop.

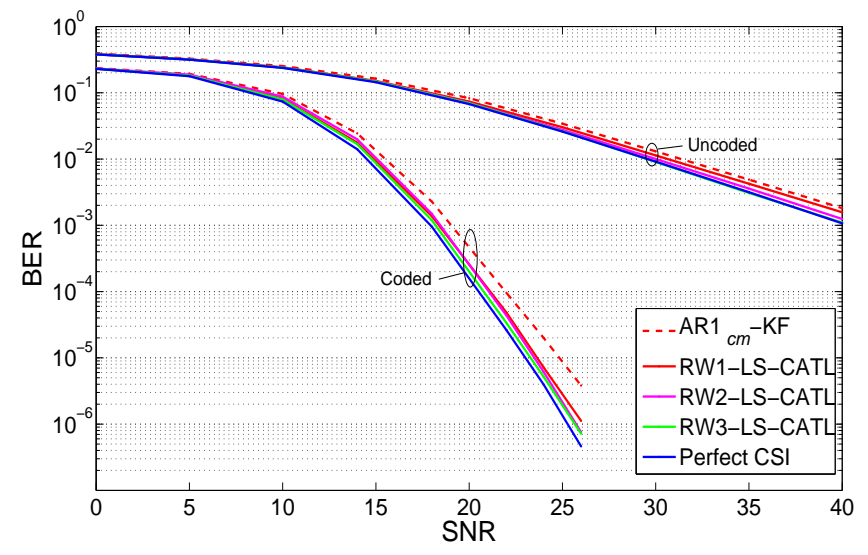

Fig. 9: BER performance for 64-QAM modulation, $f_{d} T=$ $10^{-3}, N_{p}=8$ uncoded and coded (NRNSC) $[5,7]_{8}$ scenarios, GSM channel

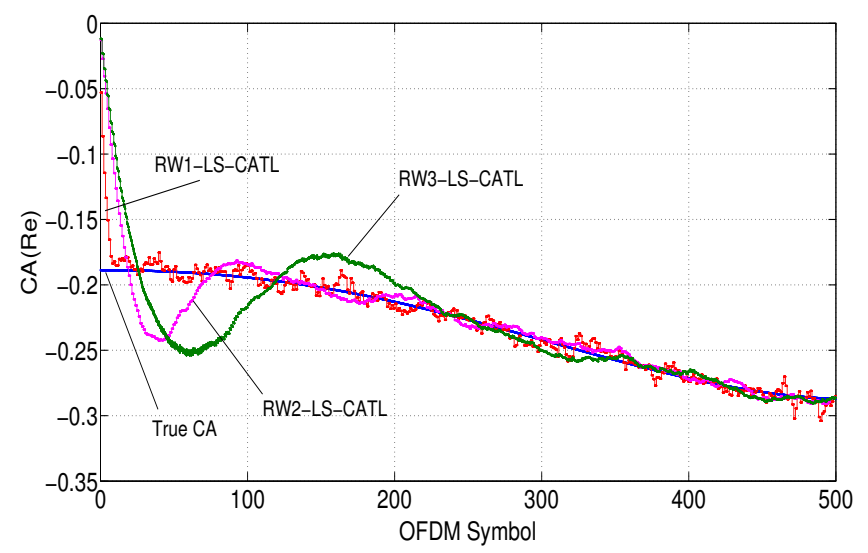

Fig. 10: Tracking performance of RW $r$-LS-CATL, $f_{d} T=$ $10^{-3}, \mathrm{SNR}=20 \mathrm{~dB}, N_{p}=16, \mathrm{GSM}$ channel

This relation has been generalised to the second order loop with $f_{d(3 \mathrm{D})} / f_{d(\mathrm{Jakes})}=\sqrt[4]{\frac{15}{8}}$, and to the third order loop with $f_{d(3 \mathrm{D})} / f_{d(\text { Jakes })}=\sqrt[6]{\frac{35}{16}}$. A performance comparison with the asymptotic Kalman filter was also provided.

\section{APPENDIX: GLOBAL OPTIMIZATION OF THE RW $r$-LS-CATL}

We start from RW3, to minimize (29), we set its partial derivatives to zero:

$$
\left\{\begin{array}{l}
\frac{\partial \sigma_{\epsilon}^{2}}{\partial f_{n} T}=\pi \sigma_{\text {Ls }}^{2} \mathcal{B}(m, \zeta)-\frac{3 S_{\alpha}}{(m \zeta)^{2}\left(f_{n} T\right)^{7}}=0, \\
\frac{\partial \sigma_{\epsilon}^{2}}{\partial m}=\pi \sigma_{\text {Ls }}^{2} f_{n} T \mathscr{D}_{m}-\frac{S_{\alpha}}{m^{3} \zeta^{2}\left(f_{n} T\right)^{6}}=0, \\
\frac{\partial \sigma_{\epsilon}^{2}}{\partial \zeta}=\pi \sigma_{\text {Ls }}^{2} f_{n} T \mathscr{D}_{\zeta}-\frac{S_{\alpha}}{m^{2} \zeta^{3}\left(f_{n} T\right)^{6}}=0
\end{array}\right.
$$


with

$$
\begin{aligned}
\mathscr{D}_{m}= & \frac{\partial \mathcal{B}(m, \zeta)}{\partial m} \\
= & \frac{m^{4} \zeta^{5}+4 m^{3} \zeta^{5}+8 m^{2} \zeta^{5}+8 m \zeta^{3}-m \zeta+2 \zeta}{2 m^{4} \zeta^{4}+8 m^{3} \zeta^{4}+8 m^{2} \zeta^{4}+4 m^{2} \zeta^{2}+8 m \zeta^{2}+2} \\
\mathscr{D}_{\zeta}= & \frac{\partial \mathcal{B}(m, \zeta)}{\partial \zeta} \\
= & \frac{2 m^{5} \zeta^{6}+16 m^{4} \zeta^{6}+32 m^{3} \zeta^{6}+16 m^{2} \zeta^{6}+\cdots}{4 m^{4} \zeta^{6}+16 m^{3} \zeta^{6}+16 m^{2} \zeta^{6}+8 m^{2} \zeta^{4}+16 m \zeta^{4}+4 \zeta^{2}} \\
& \cdots+20 m^{2} \zeta^{4}-3 m^{2} \zeta^{2}+16 m \zeta^{4}+4 \zeta^{2}-1
\end{aligned}
$$

From (47), we obtain the optimum $f_{n} T$ as a function of $m, \zeta, S_{\alpha}$ and $\sigma_{\text {LS }}^{2}$ :

$$
f_{n} T_{\text {(opt) }}=\left(\frac{3 S_{\alpha}}{\pi \mathcal{B}(m, \zeta) \sigma_{\mathbf{L S}}^{2}(m \zeta)^{2}}\right)^{\frac{1}{7}}
$$

Rewrite (48) and (49),

$$
\left\{\begin{array}{l}
m^{3} \zeta^{2} \mathscr{D}_{m} \pi \sigma_{\mathbf{L S}}^{2}\left(f_{n} T\right)^{7}-S_{\alpha}=0, \\
m^{2} \zeta^{3} \mathscr{D}_{\zeta} \pi \sigma_{\mathbf{L S}}^{2}\left(f_{n} T\right)^{7}-S_{\alpha}=0 .
\end{array}\right.
$$

Then replace $f_{n} T$ by (52), we can obtain a simplified system:

$$
\left\{\begin{array}{l}
m \mathscr{D}_{m}-\frac{\mathcal{B}(m, \zeta)}{3}=0, \\
\zeta \mathscr{D}_{\zeta}-\frac{\mathcal{B}(m, \zeta)}{3}=0 .
\end{array}\right.
$$

This system no longer depends on $\sigma_{\mathrm{LS}}^{2}$ and $S_{\alpha}$. We solve this system and obtain an optimum solution ${ }^{1} m_{\text {(opt) }}=14.3$ and $\zeta_{\text {(opt) }}=0.16$.

Similarly, for RW2, we solve the system

$$
\left\{\begin{array}{l}
\frac{\partial \sigma_{\epsilon}^{2}}{\partial f_{n} T}=\pi \sigma_{\mathbf{L S}}^{2}\left(\zeta+\frac{1}{4 \zeta}\right)-\frac{2 S_{\alpha}}{\left(f_{n} T\right)^{5}}=0, \\
\frac{\partial \sigma_{\epsilon}^{2}}{\partial \zeta}=\pi \sigma_{\mathbf{L S}}^{2} f_{n} T\left(1-\frac{1}{4 \zeta^{2}}\right)=0,
\end{array}\right.
$$

leading to $\zeta_{\text {(opt) }}=0.5$ and $f_{n} T_{\text {(opt) }}$ given in Table II.

And for RW1, we have:

$$
\frac{\partial \sigma_{\epsilon}^{2}}{\partial f_{n} T}=\pi \sigma_{\mathrm{LS}}^{2}-\frac{2 S_{\alpha}}{\left(f_{n} T\right)^{3}}=0
$$

leading to $f_{n} T_{\text {(opt) }}$ given in Table II.

\section{REFERENCES}

\section{REFERENCES}

[1] H. Shu, L. Ros, and E. P. Simon, "Complex amplitudes tracking loop for multi-path channel estimation in OFDM systems: Synthesis and extension," in 6th International Symposium on Communications, Control and Signal Processing (ISCCSP), 2014, May 2014, pp. 340-343.

[2] Y. Zhao and A. Huang, "A novel channel estimation method for OFDM mobile communication systems based on pilot signals and transformdomain processing," in IEEE 47th Vehicular Technology Conference, 1997, vol. 3, 1997, pp. 2089-2093 vol.3.

[3] M.-H. Hsieh and C.-H. Wei, "Channel estimation for OFDM systems based on comb-type pilot arrangement in frequency selective fading channels," IEEE Trans. Consum. Electron., vol. 44, no. 1, pp. 217-225, 1998.

[4] S. Coleri, M. Ergen, A. Puri, and A. Bahai, "Channel estimation techniques based on pilot arrangement in OFDM systems," IEEE Trans. Broadcast., vol. 48, no. 3, pp. 223-229, 2002.

${ }^{1}(55)-(56)$ is a multi-root system, but we keep the unique real solution.
[5] L. Ros, H. Hijazi, and E. P. Simon, "Complex amplitudes tracking loop for multipath channel estimation in OFDM systems under slow to moderate fading," ELSEVIER Signal Process., vol. 97, pp. 134-145, 2014.

[6] W. Chen and R. Zhang, "Kalman-filter channel estimator for OFDM systems in time and frequency-selective fading environment," in Proc. IEEE Intl. Conf. Acoust. Speech, Signal Process., vol. 4, 2004, pp. 377380.

[7] T. Al-Naffouri, "An EM-Based Forward-Backward Kalman Filter for the Estimation of Time-Variant Channels in OFDM," IEEE Trans. Signal Process., vol. 55, no. 7, pp. 3924-3930, 2007.

[8] M. Sohail and T. Al-Naffouri, "An EM based frequency domain channel estimation algorithm for multi-access OFDM systems," ELSEVIER Signal Process., vol. 90, no. 5, pp. 1562 - 1572, 2010.

[9] B. Yang, K. B. Letaief, R. S. Cheng, and Z. Cao, "Channel estimation for OFDM transmission in multipath fading channels based on parametric channel modeling," IEEE Trans. Commun., vol. 49, no. 3, pp. 467-479, 2001.

[10] M. R. Raghavendra, S. Bhashyam, and K. Giridhar, "Exploiting hopping pilots for parametric channel estimation in OFDM systems," IEEE Signal Process. Lett., vol. 12, no. 11, pp. 737-740, 2005.

[11] C. Komninakis, C. Fragouli, A. Sayed, and R. Wesel, "Multi-input multi-output fading channel tracking and equalization using Kalman estimation," IEEE Trans. Signal Process., vol. 50, no. 5, pp. 1065-1076, May 2002.

[12] S. Hwang, J. Um, M. Song, C. Kim, H. Park, and Y. Kim, "Design and verification of IEEE 802.22 WRAN physical layer," in 3rd International Conference on Cognitive Radio Oriented Wireless Networks and Communications, 2008.

[13] H. Shu, E. P. Simon, and L. Ros, "Third-order kalman filter: Tuning and steady-state performance," IEEE Signal Process. Lett., vol. 20, no. 11, pp. 1082-1085, 2013.

[14] H. Hijazi and L. Ros, "Joint data QR-detection and Kalman estimation for OFDM time-varying Rayleigh channel complex gains," IEEE Trans. Commun., vol. 58, no. 1, pp. 170-178, Jan. 2010.

[15] E. P. Simon, L. Ros, H. Hijazi, and M. Ghogho, "Joint Carrier Frequency Offset and Channel Estimation for OFDM Systems via the EM Algorithm in the Presence of Very High Mobility," IEEE Trans. Signal Process., vol. 60, no. 2, pp. 754-765, Feb. 2012.

[16] S. Ghandour-Haidar, L. Ros, and J.-M. Brossier, "On the use of firstorder autoregressive modeling for Rayleigh flat fading channel estimation with Kalman filter," Signal Processing, vol. 92, no. 2, pp. 601 606, 2012.

[17] L. Ros, S. Ghandour-Haidar, and J.-M. Brossier, "Sur l'utilisation des filtres du premier-ordre pour l'estimation d'un canal radio-mobile de Rayleigh à évanouissement plat," in XXIIIème colloque GRETSI (GRETSI 2011), Bordeaux : France, 2011.

[18] R. Gerzaguet, L. Ros, J.-M. Brossier, S. Ghandour-Haidar, and F. Belvèze, "Self-adaptive stochastic rayleigh flat fading channel estimation," in IEEE International Conference on Digital Signal Processing (DSP), 2013.

[19] L. Ros and E. P. Simon, "Second-order modeling for Rayleigh flat fading channel estimation with Kalman Filter," in 17th International Conference on Digital Signal Processing (DSP), 2011, pp. 1-6.

[20] L. Ros, H. Hijazi, and E. P. Simon, "Paths complex gain tracking algorithms for ofdm receiver in slowly-varying channels," in Proc. Intl. Symp. Commun. Control, Signal Process., Mar. 2010, pp. 1-6.

[21] L. Lindbom, A. Ahlen, M. Sternad, and M. Falkenstrom, "Tracking of time-varying mobile radio channels. II. A case study," IEEE Trans. Commun., vol. 50, no. 1, pp. 156-167, Jan 2002.

[22] Q. Nasir, "Wireless Channel Tracking Based on Optimum Predictive LMS," Wireless Personal Communications, vol. 48, no. 4, pp. 511-519, 2009.

[23] A. Patapoutian, "On phase-locked loops and Kalman filters," IEEE Trans. Commun., vol. 47, no. 5, pp. 670-672, May, 1999.

[24] H. Shu, L. Ros, and E. P. Simon, "Third-order complex amplitudes tracking loop for slow flat fading channel online estimation," IET Communications, vol. 8, pp. 360-371(11), February 2014. [Online]. Available: http://digital-library.theiet.org/content/journals/10.1049/ietcom.2013.0316

[25] — " "Third-order complex amplitudes tracking loop for slow fading channel estimation," in 19th International Conference on Telecommunications (ICT), April, 2012, pp. 1-6.

[26] R. H. Clarke and W. L. Khoo, "3-D mobile radio channel statistics," IEEE Trans. Veh. Technol., vol. 46, no. 3, pp. 798 -799, aug 1997. 
[27] Y. Rosmansyah, S. Saunders, P. Sweeney, and R. Tafazolli, "Equivalence of flat and classical Doppler sample generators," Electronics Letters, vol. 37, no. 4, pp. 243-244, Feb 2001.

[28] H. Shu, L. Ros, and E. P. Simon, "Simplified random-walk-model-based kalman filter for slow to moderate fading channel estimation in ofdm systems," IEEE Trans. Signal Process., vol. 62, no. 15, pp. 4006-4017, Aug 2014.

[29] K. Muralidhar, "Equivalence of VSSO and VSVO Kalman Channel Estimators in Doubly-Selective OFDM Systems - A Theoretical Perspective," IEEE Signal Process. Letters, vol. 18, no. 4, pp. 223-226, April 2011.

[30] M. Karthik and D. Sreedhar, "VSSO Kalman-usage of Barhumi's pilot pattern for channel estimation in MIMO-OFDM systems," Electronics Letters, vol. 48, no. 14, pp. 883-884, July 2012.

[31] K. Muralidhar and K. H. Li, "A Low-Complexity Kalman Approach for Channel Estimation in Doubly-Selective OFDM Systems," IEEE Signal Process. Letters, vol. 16, no. 7, pp. 632-635, July 2009.

[32] K. Muralidhar and D. Sreedhar, "Pilot Design for Vector State-Scalar Observation Kalman Channel Estimators in Doubly-Selective MIMOOFDM Systems," IEEE Wireless Commun. Letters, vol. 2, no. 2, pp. 147-150, April 2013.

[33] W. Jakes, Microwave Mobile Communications. New-York: John Wiley, 1974.

[34] H. Hijazi and L. Ros, "Bayesian Cramera-Rao Bounds for complex gain parameters estimation of slowly varying Rayleigh channel in OFDM systems," ELSEVIER Signal Processing, vol. 89, no. 1, pp. 111 - 115, 2009.

[35] R. Roy and T. Kailath, "ESPRIT-estimation of signal parameters via rotational invariance techniques," IEEE Trans. on Acoustics Speech and Signal Processing, vol. 37, no. 7, pp. 984-995, 1989.

[36] L. Ros, H. Hijazi, and E. P. Simon, "Complex amplitudes tracking loop for multipath slow fading channel estimation in OFDM systems," https://hal.archives-ouvertes.fr/hal-00687821, Tech. Rep., Apr. 2012. [Online]. Available: https://hal.archives-ouvertes.fr/hal-00687821

[37] European Telecommunications Standards Institute, European Digital Cellular Telecommunication System (Phase 2), Radio Transmission and Reception, GSM 05.05, vers. 4.6.0. 